NABA TECHNICAL MEMORANDUM

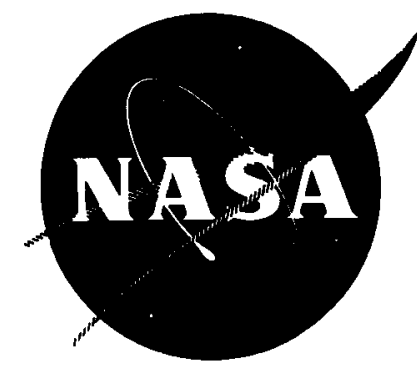

NASA TM X- 52189

$\frac{1}{\infty}$
1
$\frac{1}{2}$
$\frac{1}{2}$
$\frac{1}{2}$
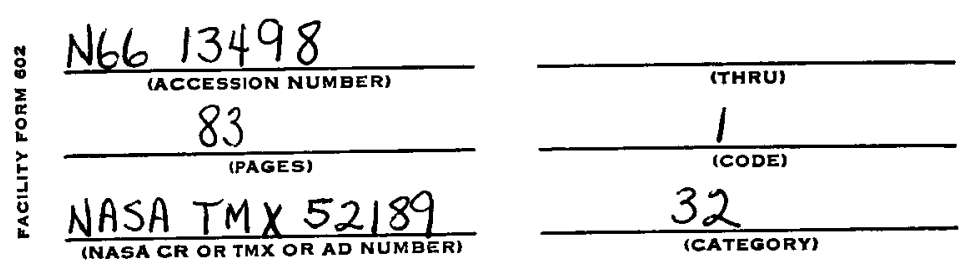

INTERFACES BETWEEN FATIGUE, CREEP, AND FRACTURE

by S. S. Manson

Lewis Research Center

Cleveland, Ohio

Special Lecture presented at the

International Conference on Fracture,

- Sendai, Japan, September 14, 1965

NATIONAL AERONAUTICS AND SPACE ADMINISTRATION - WASHINGTON, D.C. 1966

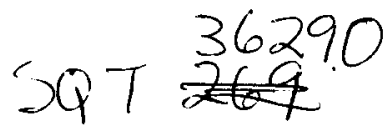




\section{INTERFACES BETWEEN FATIGUE, CREEP, AND FRACTURE}

by S. S. Manson

\section{Lewis Research Center}

Cleveland, Ohio

Special Lecture presented at the International

Conference on Fracture, Sendai, Japan,

September 14, 1965

Published in the International Journal of Fracture Mechanics, March 1966, and to be published in the Proceedings of the International Conference on Fracture

NATIONAL AERONAUTICS AND SPACE ADMINISTRATION 


\section{INTERFACES BETWEEN FATIGUE, CREEP, AND FRACTURE \\ by S. S. Manson \\ Lewis Research Center \\ National Aeronautics and Space Administration \\ Cleveland, Ohio \\ INTRODUCTION}

In attempting to decide upon the content of this lecture, I deliberated between the choice of providing a summary of the existing literature on the subject assigned to me by the Organizing Committee for the Conference, and the alternative of presenting to you some current thoughts on the subject--thoughts that had not previously matured to the point of publication. I chose the latter alternative, realizing that many of my remarks would have to be regarded as speculative rather than being based on an extensive experimental foundation. The intent is to stimulate thought and perhaps further work in the areas of research to be discussed rather than to present conclusive evidence of their validity.

The subject assigned to me was "Interfaces Between Fatigue, Creep, and Fracture". I shall interpret "interfaces" to imply that concepts used in two or more disciplines have to be brought together in order to arrive at a conclusion. In attempting to fulfill the requirement, my lecture will deal with three subjects. The first is an interface between fatigue and fracture which will be concerned with the fatigue problem of quasi-brittle materials; the second will attempt to bring together creep and fatigue by considering fatigue in the creep range at elevated temperatures, and the third will deal with cumulative fatigue damage. Since I shall consider cumulative fatigue damage as a process of initiating and 
propagating a crack, it may properly be considered as a second interface between fatigue and fracture.

\section{Background}

Some of the concepts that will be used are contained in a paper presented to the Society for Experimental Stress Analysis in 1964 (ref. 1). Before considering the various interfaces it would be instructive to review several points of this earlier paper. At the outset it is very important to emphasize that the conclusions drawn are specificaliy applicable only to certain types of specimens. Extending them to specimens of different geometries or to engineering structures may involve new and difficult procedures. The type of specimen used to obtain most of the data upon which the concepts presented here are based is shown in figure 1 . It is unnotched and has an hour-glass shape with a minimum diameter of $I / 4$ inch. In most of our studies on such specimens, load is applied aiternately in tension and compression, cycling about a zero mean stress and a zero mean strain. Usually the strain is measured in a transverse direction. A few of our tests (ref. 2) have been conducted on specimens with notches as shown in figure 2 . The notch depth is approximately 0.010 inch; this dimension is especially significant since it enters into the determination of the "engineering size crack" discussed in reference 2. Specimens with notches of this depth with concentration factors of 2 or 3 have been tested in our program. For some of our tests conventional R. R. Moore bending specimens have also keen used. It must be recognized, therefore, that the test results which will be referred to were obtained on rather small specimens. The specimens usually had carefully machined surfaces, and in most cases were tested in air in the absence of pernicious environments. 
In interpreting the results of our low-cycle fatigue tests, it is common to regard cyclic life as being governed largely by the strain range to which the test section is subjected. Figure 3 illustrates the concept we have applied to the plot of total strain range against the number of cycles to failure. Failure is defined here as separation of the specimen into two parts. The total strain range which is given by the curved line has been separated into its two components, plastic and elastic. It is evident from the figure that on a log-log plot of strain versus number of cycles to failure, both the elastic and the plastic components are assumed to be represented by straight lines. The linearity of a logarithmic plot of plastic strain range versus life has been well accepted in the literature on fatigue in recent years. We first proposed this linearity in 1952 (ref. 3) in connection with the thermal fatigue of turbine buckets. The concept was also proposed independently by Coffin (ref. 4) and much research information has been compiled by numerous authors to verify that such a linear relationship exists between plastic strain and life. The -0.6 slope shown in figure 3 for the plastic line is a reasonable average representation of most of the materials we have tested. It is common to assume that the plastic line has a universal slope of -0.5 . However, there is really no basic conflict with the -0.6 slope shown in figure 3 since in most cases where -0.5 has been proposed the life has been that required to generate a crack rather than that required to separate the specimen into two parts. In reference 1 we have attempted to demonstrate that the difference in slopes is directly attributable to the difference in definition of fatigue life.

It is evident from figure 3 that the elastic line is also assumed to 
be straight. This observation is of more recent origin (refs. 5-8). The slope in this case is on the order of -0.12. As implied in the figure, both the plastic and the elastic components can be estimated from measurements of properties obtained in a simple tensile test. Thus from a knowledge of the ductility of the material, it is possible to determine one point on the plastic line, and from the ultimate tensile strength and elastic modulus one point on the elastic line may be determined. Then, by means of the assumed universal slopes both components can be drawn. The equation for total strain range as a function of life is given at the top of figure 3 .

The validity of the procedure shown in figure 3 for estimating the fatigue properties from a knowledge of the static tensile properties has been demonstrated in reference 1 for 29 materials. Comparison between estimated and observed fatigue lives for 10 additional materials that have since been tested are shown in figure 4 . The circles represent the experimental data; the curves that are shown in connection with the data points for each material represent the predictions based on the method of universal slopes just discussed. Very good agreement was obtained between the estimated and observed lives for all the materials shown.

Additional confidence in the approximate validity of the method of universal slopes has been gained by considering its usefulness in determining from static tensile properties alone whether the material will be strain hardening or strain softening under cyclic conditions. This subject is discussed in appendix $A$ and illustrated in figure 5(a). Results are presented for 30 materials in the bar graph of figure 5(b). According to the predic- 
tions of the analysis based on the method of universal slopes all the bars that lie above the horizontal axis in this figure should be cyclic strain hardening, while all those lying below the horizontal axis should be strain softening. That the predictions agreed well with the experimental observations is indicated by the shading of the bars.

We have developed a considerable degree of confidence in the method of universal slopes for predicting the fatigue lives at room temperature and in a later section of this paper the concept is further discussed in connection with predictions of fatigue properties at high temperature.

\section{TNTERFACE BETWEEN FATIGUE AND FRACTURE}

Let us now examine the model of figure 3 in relation to the determination of the fatigue properties of a quasi-brittle material. We note that in estimating the fatigue properties on the basis of this model only the properties ordinarily obtained in a conventional tensile test, such as ductility, ultimate tensile strength and elastic modulus are used. From the point of view of fracture mechanics it might be considered that such properties as fracture toughness should be involved. After all, is not the final cycle of the fatigue test one which terminates in fracture due to the development of a crack of critical size for the stress range to which the material is subjected? Why, then, should fracture toughness not enter into the representation of the fatigue behavior? The primary reason is that in the testing of small unnotched specimens most of the fatigue life is taken up in initiating a crack and in the early stages of propagation. The later development of the crack from a moderate size to critical size takes only a small fraction of the total fatigue life because the rate of crack propaga- 
tion per cycle at this stage is relatively high . Thus the fraction of. the total life involved in this stage is quite small and variations in this fraction may be indistinguishable within the scatter of the fatigue life data. In addition, the size of the $1 / 4$ inch diameter fatigue specimen does not permit much variation in critical crack sizes. The toughness of most engineering materials is sufficiently high that failure of such a specimen at a net section stress less than the yield strength cannot occur. Since the conditions for a linear elastic fracture mechanics toughness test are rarely realized in fatigue testing of small, smooth specimens, it should occasion no surprise that the fatigue life of a small specimen can be predicted without reference to the fracture toughness of the material. In order to determine wherein the importance of fracture toughness lies, we must, therefore, resort to materials of low toughness, so that brittle fracture will be manifest even in small specimens. We must also start with a notched specimen, such as the one shown in figure 2, to promote the process of crack initiation early in the cyclic loading process. As a vehicle for discussing this interface between fatigue and fracture we will therefore choose to treat the behavior of notched specimens of quasibrittle materials which have relatively low fracture toughness. Before doing so, however, it is necessary to establish a model for crack initiation and for crack growth, as well as to postulate a condition for brittle fracture of specimens of the type shown in figure 1 . Here it is important to emphasize the speculative feature discussed in the INTRODUCTION. The models to be discussed are not based on abundant or conclusive evidence, but they may be helpful as a start. 
Ductile Versus Brittle Behavior

Before discussing brittle behavior in fatigue it is important to know how a highly ductile material behaves under the same conditions. We shall consider copper, a typical failure surface of which is shown in figure 6 . Each of the progressive striations is caused during one cycle of loading. These striations extend almost across the entire cross section, indicating that the crack depth at failure can be regarded as almost the entire diameter of the specimen. On the other hand, the spacing of the striations becomes progressively larger as the crack gets larger. Thus, whether failure is considered to occur when the crack length is equal to the diameter, or whether it is taken as a large fraction of the diameter (say 60 to $90 \%$ ) has relatively small effect on the determined number of cycles to failure. This fact of insensitivity of cyclic life to crack length at fracture will later be used in postulating a universal crack growth law for ductile materials.

It is important to recognize that the spacing of the striations after the crack has penetrated to an appreciable depth (above approximately $0.003 \mathrm{in.)}$ depends to some extent on the manner in which the fatigue test is carried out. Our tests are conducted by maintaining a constant diametral strain range, which implies an almost constant axial strain range (depending on the degree of hardening or softening during the test). This diametral strain is monitored by means of optical strain gages which involve the use of knife edges that bear on the specimen at the test section (ref. 5). Once an appreciable crack develops, the interpretation of the readings of the diametral gage becomes difficult. 
Several alternatives are possible for continuing the test once the crack is of macroscopic size. One approach is to continue to monitor the apparent transverse strain as if the crack were absent; another is to remove the gage and to continue the test maintaining either the load range or the end-displacement achieved at the time of gage removal. Crack growth rates may depend appreciably on which of the procedures is followed, and discrepancies in results based on striation measurements may, in fact, be due to differences in techniques used by different investigators. In most of our tests we have preferred to remove the gage and maintain enddisplacement. This approach would appear to be more representative of what happens in the region of a small crack in a large body which is, in the main, undergoing elastic loading; the displacements at the boundary of the region of the small crack tend to be governed by the elastic strains in the structure, which change little as the crack grows. There is, however, an additional reason for preferring the fixed end-displacement procedure. When the load range is maintained constant, the net section stress progressively increases as the crack grows larger; the same phenomenon is observed, under certain conditions, when the nominal diametral strain range is held constant. The final fracture area will therefore depend to a considerable extent on the loads imposed. When end displacement is maintained constant, however, the load progressively decreases, and the crack may gradually penetrate the entire cross-section before the specimen separates into two parts. Highly ductile materials, such as copper, will then show by striations a characteristic of progressive crack growth as in figure 6 . It thus becomes possible to assume that in a completely ductile material 
the crack depth at failure is approximately constant regardless of material and strain range, rather than being material and strain dependent, as would be the case if load range were maintained constant. This assumption will be very useful in the development of the analytical procedure to be described.

In contrast to the behavior of a highly ductile material, a material of low notch toughness may develop a failure cross-section such as shown in figure 7, even if the test is conducted by maintaining end-displacement amplitude once the crack starts. In this case the final fracture took place well before the crack had penetrated the entire cross section. Such brittle fracture is due to the attainment of a critical combination of crack depth and applied load. Thus, although the load decreases while the crack grows deeper, such a critical combination of crack depth and load can develop when the fracture toughness of the material is low enough. The procedure whereby the critical combination can be determined will now be discussed.

\section{Crack Initiation and Crack Growth}

Before discussing the fracture condition of a quasi-brittle material as a consequence of the development of a fatigue crack within its test section, It is necessary to postulate laws for crack initiation and for crack growth. An attempt at the treatment of this problem has already been made in reference 1. Further analysis of striation data has made possible a tentative revision and extension of the hypothesis there stated.

In reference $I$ it was postulated that if the life of a specimen was $\mathrm{N}_{\mathrm{f}}$ cycles, the period of crack propagation could be taken as $2.5 \mathrm{~N}_{\mathrm{f}}^{2 / 3}$ 
cycles. The period of crack propagation was not clearly defined because of the lack of extensive data, but, as indicated in reference I, the exjression referred to the number of cycles to advance the crack from a depth of approximately 0.002 to 0.003 inch to a crack size for failure. For the present analysis the treatment was generalized to consider crack growth from a stage earlier than when a depth of 0.002 to 0.003 inch had developed. To establish a more useful working hypothesis the striation spacings for a number of copper and silver specimens were examined to determine whether a reasonable crack growth law could be established. Equation (I) appears to represent the available limited data:

$$
\frac{d l}{d N}=f\left(\Delta \epsilon_{p}\right) l
$$

where

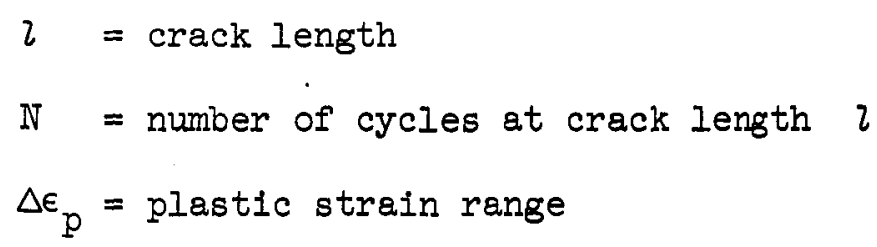

The expression $f\left(\Delta \epsilon_{p}\right)$ is shown in equation (I) as a general function of plastic strain range. The exact functional form is not important in the following development since the entire term becomes cancelled during the mathematical treatment; however, comments as to the form of the function are included in appendix $B$.

Equation (1) may now be integrated on the assumption that $\Delta \epsilon_{p}$, and therefore, $f\left(\Delta \epsilon_{p}\right)$, is a constant (such an assumption is, of course, only: an approximation since after the crack starts the test becomes one of constant-end-displacement, so that $\Delta \epsilon_{p}$ probably changes as the crack 
advances) giving the following equation

$$
\text { In } \frac{2}{l_{0}}=f\left(\Delta \epsilon_{p}\right)\left(\mathbb{N}-N_{0}\right)
$$

where $\tau_{0}$ and $N_{O}$ are any corresponding values of crack length and cycles. To obtain the fracture condition we substitute $\imath=l_{f}$ and $N=N_{f}$. Thus

$$
\ln \frac{l_{f}}{l_{0}}=f\left(\Delta \epsilon_{p}\right)\left(N_{f}-N_{0}\right)
$$

Dividing equation (2) by equation (3) we get

$$
\frac{\ln \frac{l}{l_{0}}}{\ln \frac{l_{f}}{l_{0}}}=\frac{N-N_{0}}{N_{f}-N_{0}}=\frac{\Delta N}{(\Delta N)_{f}}
$$

In order to attach a very simple significance to equation (4) we postulate that both $l_{0}$ and $l_{f}$ can be taken as constant for all materials and strain levels. Choosing $l_{0}$ as a constant is perfectly admissible since it is an arbitrary constant of integration, and the cholce of any specific value merely defines the value of life, $\mathrm{N}_{\mathrm{O}}$, assoclated with it. We shall define $l_{0}$ as the lowest value of $l$ for which equation (1) becomes valid as the crack propagation law, and assume for the present that this value is independent of material and strain range.

The choice of $l_{f}$ as a constant has already been justified earlier in this section in connection with the discussion of figure 6 . By selecting an arbitary value of $l_{f}$ we are essentially defining $\mathbb{N}_{f}$ as the number of cycles required to achieve this crack length $z_{f}$ instead of the number of cycles required to separate the specimen into two halves. As already 
noted, the striations become so coarsely spaced once the crack extends beyond the center of the specimen that the percentage difference between the number of cycles required to generate a crack deeper than half the specimen and that required to break the specimen is very small for all practical values of life.

Examination of equation (4) now shows that since $\imath_{0}$ and $\imath_{f}$ are constants, the left side of the equation depends only on $\tau$. The right hand side of the equation, which is the fraction of the total crack propagation period required to propagate the crack to a length $l$, depends, therefore, only on the value of 2 . Thus a single curve should be able to express $l$ in terms of $\Delta \mathrm{N} /(\Delta \mathrm{N})_{f}$. As discussed in appendix $B$, the expression for $(\Delta \mathrm{V})_{f}$ is taken as $N_{f}$ in the range of $N_{f}$ up to 730 cycles, and as $14 \mathrm{~N}_{f} \mathrm{O} \cdot 6$ for $\mathrm{N}_{f}$ above 730 cycles. Since $N_{0}$ can be determined as $N_{f}-(\Delta N)_{f}$, both the numerator and denominator in the expression $\Delta \mathrm{V} /(\Delta \mathrm{V})_{f}$ can be determined. (A graphical relation between $N_{0},(\Delta N)_{f}$, and $\mathbb{N}_{f}$ is shown in fig. 8.) The universal curve between $\tau$ and $\Delta \mathrm{N} /(\Delta \mathrm{N})_{\mathrm{f}}$ can then be determined as a compromise of available data on crack growth as determined from striation measurements. Based on our limited data obtained to date, we have chosen the following equation as a reasonable tentative approximation:

$$
\frac{\Delta V}{(\Delta V)_{f}}=\log _{10} 15.32^{1 / 4}
$$

where $(\Delta N)_{f}$ is taken as $N_{f}$ for $N_{f}<730$, and $14 N_{f}^{0.6}$ for $N_{f}>730$. This subject is further discussed in appendix $B$.

To determine the significance of equation (5) for experimental observations of crack growth, this equation is depicted in figure 9 on a semiIogarithmic coordinate system, and is a straight line. The intercept of 
the line with the abscissa $\Delta V /(\Delta V)_{f}=0$, is $l_{0}=1.8 \times 10^{-5}$ inch, and the intercept with the value $\Delta \mathrm{N} /(\Delta \mathrm{N})_{f}=1$ is 0.18 inch, implying that the crack length at failure is 0.18 inch.

Some support for the validity of equation (5) as a representation of the crack growth process for all ductile materials can be seen in figure 9 by considering the value of $\Delta \mathrm{N} /(\Delta \mathrm{N})_{f}$ for $l=0.013$ inch. This value of crack depth has special significance in relation to the behavior of notched specimens such as shown in figure 2 which have a machined notch depth of 0.010 inch. The experimental program (ref. 2) on such specimens involved cycling until a detectable crack was observed at the root of the notch, implying a crack length along the surface of approximately 0.010 inch and a depth of approximately 0.003 inch. Now, as discussed in reference 2 , the analysis of the crack growth at the root of the notch is quite complicated. While the crack is very small its growth is influenced largely by the local strain at the root of the machined notch, and its effective length is that measured from the root of the machined notch to the end of the crack. At a later stage the situation is better represented as one in which the crack is equal in size to the actual crack plus the notch depth, and the nominal strain field is that of an unnotched specimen. The crack depth at which the transition occurred was referred to in reference 2 as an "engineering size crack", and was assumed to occur when the crack could be visually detected, or when it was approximately 0.003 inch from the root of the machined notch. At this transition, then, the effective crack depth becomes $0.010+0.003=0.013 \mathrm{inch}$, and the remaining life after such a crack depth has been reached in a smooth specimen should be the same as when a 
depth of 0.003 inch is reached in a notched specimen (fig. 2). According to figure 9 , the value of $\Delta N /(\Delta N)_{f}$ for a value of $l=0.013$ inch for an unnotched specimen is 0.715 , so that the fraction of the total propagation period, $(\Delta N)_{f}$, required to extend the crack from a depth of 0.13 inch to a depth of 0.18 inch is $1-0.715=0.285$. Thus, since $\left(\Delta \mathrm{N}_{f}\right)=14 \mathrm{~N}_{\mathrm{f}}^{\mathrm{O}}{ }^{6}$, the value of $N$ between the time the crack grows from a size of 0.013 inch to failure is $0.285 \times 14 \mathrm{~N}_{\mathrm{f}}^{0.6}=4 \mathrm{~N}_{\mathrm{f}}^{0.6}$.

Figure 10 shows the results of the tests on notched specimens of 5 materials as designated. The ordinate is the number of cycles sustained by the specimen from the time detectable cracking occurred at the root of the notch to the time final specimen separation occurred at the test section. The abscissa is the cyclic life sustained by a smooth specimen under the nominal average strain range to which the notched specimen is subjected. It is seen that good agreement exists between the experimental data and the prediction $\triangle \mathrm{N}=4 \mathrm{~N}_{\mathrm{f}}^{0.6}$.

Returning to consideration of figure 9 it may be seen that the figure provides, in fact, an indication of the crack propagation period for any arbitrary criterion of crack initiation. For example, if optically-unaided detectable cracking on the surface (implying a depth of about 0.003 in.) is taken as the criterion for cracking of an unnotched specimen, figure 9 would indicate a crack propagation period of $6.2 \mathrm{~N}_{f}^{0.6}$, using the calculation rationale previously described for $l=0.013$ inch.

It should be emphasized that the above discussion in which the value of $(\Delta N)_{f}$ is taken as $14 N_{f}^{0.6}$ is valid only for $N_{f}>730$ cycles. For lower lives $(\Delta N)_{f}=N_{f}$, and all the numbers cited above must be correspond- 
ingly amended. Thus, for example, after the crack has reached a depth of 0.003 inch the remaining number of cycles before failure occurs would be $0.443 N_{f}$, and after the crack has reached 0.013 inch the remaining number of cycles before failure occurs would be $0.285 \mathrm{~N}_{\mathrm{f}}$ :

Fracture Condition

Equation (5) and figure 9 represent the hypothesized relation for crack growth (and implicitly, crack initiation) for a completely ductile material. Quasi-brittle materials, which fracture well before the crack has progressed across the entire test section will be assumed to follow the same crack initiation condition, and the same crack propagation condition until the critical crack size is reached, at which point brittle fracture will interrupt the crack growth process. It is now necessary, therefore, to establish a fracture condition for specimens of the geometry shown in figures' 1 or 2 .

Only a few tests have been conducted thus far aimed at the determination of a fracture condition for the small circular geometry of the conventional fatigue specimen. The results of these tests are shown in figure 11. Specimens of AISI 52100 steel were machined with the notch geometry of figure 2 having an elastic stress concentration factor of 2.0 . These specimens were hardened and tempered at various temperatures to achieve several fracture toughness levels $\mathrm{K}_{\mathrm{Ic}} \cdot$ The opening-mode fracture toughness $\mathrm{K}_{\text {Ic }}$ value for each temper was measured using the techniques of reference 9 on specimens of $1 / 2$ inch square section in which fatigue cracks had been introduced. These fracture toughness values varied from 17 to $35 \mathrm{ksi}-\sqrt{\mathrm{in} .}$, as listed in figure 11. The round notched specimens 
(fig. 2) of similarly heat-treated material were then fatigue-tested at various strain levels. Observations were made of the stress amplitude $\sigma$, the number of cycles to failure, and the depth of penetration of the crack at failure. In determining the crack depth, the depth of the 0.010 inch machined notch was included, and these crack lengths at failure were plotted against $K_{I c} / \sigma$ as shown in figure 11. Linear elastic fracture mechanics theory would predict that a logarithmic plot of $l_{f}$ versus $\mathrm{K}_{\mathrm{Ic}} / \sigma$ should produce a straight line of slope 2. The data lent themselves readily to such a correlation, the equation of the straight line of figure 11 being

$$
\eta_{f}^{\prime}=\left(\frac{K_{I c}}{\sigma}\right)^{2} \text { or } K_{I c}=\sigma \sqrt{\eta_{f}^{\prime}}
$$

where $i_{f}$ is the crack length at fracture for the quasi-brittle material (in contrast to the designation $i_{f}$ for the failure crack length for completely ductile behavior, which is taken as 0.018 in. for all materials). While equation (6) is manifestly derived for only one material, and for a limited range of $\mathrm{K}_{\mathrm{Ic}}$, its remarkable simplicity makes it attractive for general use. Further data are needed to justify its use for applications beyond the illustrations cited here. Equation (6), or its more general equivalent when determined, then provides an expression for the crack depth at failure for a material of known value of $K_{I c}$ cycled at a known stress amplitude $\sigma$. If the expression yields a value of $\eta_{f}^{\prime}$ greater than 0.18 inch it may be assumed that completely ductile behavior (according to the definition previously cited) will characterize the material at test conditions involved. If the value of $l_{f}^{\prime}$ is less than 
0.18 inch a quasi-brittle failure will occur when the crack depth becomes equal to $\tau_{f}^{\prime}$.

Application to Unnotched Specimens

When a fatigue specimen behaves in a completely ductile manner throughout a test the fatigue life $N_{f}$ can be predicted from its relation to the strain range and the tensile properties of the material as given by the method of universal slopes (fig. 3). It is of interest to predict the life $\mathbb{N}_{f}^{\prime}$ of a specimen which fails prematurely by brittle fracture and to see to what extent the ratio $\mathrm{N}_{f} / \mathrm{N}_{f}^{\prime}$ depends upon $\mathrm{K}_{I c}$. This is accomplished by use of equations (5) and (6). Because of the double condition placed on $(\Delta \mathrm{V})_{f}$ in equation (5) it is necessary to examine separately the two cases $\mathrm{N}_{f}<730$, and $\mathrm{N}_{\mathrm{f}}>730$.

Case I: $N_{f}<730$ cycles. For this case $N_{O} \approx 0$, and $N=N_{f}^{\prime}$, and $\left(\Delta N_{f}=\mathbb{N}_{f^{*}}\right.$. By equations (5) and (6)

$$
\Delta \mathrm{N}=(\Delta \mathrm{N})_{f} \log _{10^{15}} 152^{1 / 4}=(\Delta \mathrm{V})_{f} \log _{10^{15}} 15 \sqrt{\frac{K_{I c}}{\sigma}}
$$

Therefore

$$
N_{f}^{\prime}=N_{f} \log _{10} 15 \cdot 3 \sqrt{\frac{K_{I c}}{\sigma}}
$$

Case II: $N_{f}>730$ cycles. Here $(\Delta N)_{f}=14 N_{f}^{0.6}, N_{0}=N_{f}-(\Delta N)_{f}=$ $N_{f}-14 N_{f}^{0.6}$. By equations (5) and (6)

$$
\Delta \mathrm{N}=\left(\Delta \mathrm{N}_{\mathrm{f}}\right)_{\log _{10} 15.3} \sqrt{\frac{\mathrm{K}_{\mathrm{Ic}}}{\sigma}}=14 \mathrm{~N}_{\mathrm{f}}^{0.6} \log _{10} 15.3 \sqrt{\frac{\mathrm{K}_{\mathrm{Ic}}}{\sigma}}
$$

But $N_{f}=N_{0}+\Delta N$, therefore

$$
N_{f}^{\prime}=N_{f}-14 N_{f}^{0.6}+14 N_{f}^{0.6} \log _{10} 15.3 \sqrt{\frac{K_{I c}}{\sigma}}
$$


The relationships among $\mathrm{N}_{f}, \mathrm{~N}_{f}^{\prime}$, and $\mathrm{K}_{\mathrm{Ic}} / \sigma$ expressed by equations (7) and (8) are shown graphically in figure 12. It can be seen from figure 12 that at the very low cyclic lives and very low values of $\mathrm{K}_{\mathrm{Ic}} / \sigma$, $\mathrm{N}_{f}$ may be appreciably larger than $\mathrm{N}_{f}^{\prime}$. As the life is increased the effect becomes smaller and smaller. This result is to be expected on the basis of the relative duration of the crack initiation and crack propagation periods. For the low cyclic lives the entire life consists of crack propagation. Interrupting the crack propagation by premature brittle failure before the crack has progressed across the entire section, therefore, reduces the life appreciably. Even so the loss of life is only of the order of 50 percent or less for practical ranges of $\mathrm{K}_{\mathrm{Ic}} / \sigma$, and this amount of life reduction might not be clearly distinguishable from the data scatter common in fatigue measurements.

At the higher cyclic lives the effect becomes very small indeed, and is surely to be hidden in data scatter. The reason for the small effect at the higher cyclic lives lies in the relative unimportance of the crack propagation phase. Most of the life is consumed in the crack initiation phase which is unaffected by the fracture toughness, according to the approach adopted here. The only effect of the fracture toughness is to cause premature fracture at some critical crack depth during propagation of the crack. Since the crack propagation phase is a small part of the total life, removing even most of it does not have a large effect on total Iife.

Thus it can be seen why fracture toughness does not enter as a major variable in the estimation of fatigue life of small unnotched specimens. 
This observation has already been made in reference 1; the above discussion and figure 12 serve mainly to make the explanation more quantitative. Application to Notched Specimens

While fracture toughness is relatively unimportant in affecting the life of small unnotched specimens, the effect becomes more important when a notched specimen is used for material evaluation. The strain concentration at the notch root causes the crack to develop at a relatively early stage in the life of the specimen compared to an unnotched specimen, making the crack propagation period a larger percentage of the total life. Since low fracture toughness serves to cut short the crack propagation period, the effect of low fracture toughness would be expected to be of greater importance in such notched specimens than in unnotched specimens. The method of computation again depends predominantiy on the use of equations (5) and (6), or their graphical equivalents, figures 9 and 11 . We shall illustrate the approach by referring again to the AISI 52100 steel tempered at $600^{\circ} \mathrm{F}$ for which the value of $K_{\text {Ic }}$ was $17 \mathrm{ksi}-\sqrt{\mathrm{in} .}$ (fig. 11 ). An illustrative computation is presented in appendix $\mathrm{C}$; here we shall indicate only the basis of the method and the results of the computations.

In figure $13(a)$ the continuous line represents the strain cycling fatigue behavior for unnotched specimens determined by the method of universal slopes. It is assumed that this curve would be obtained for the material having the measured tensile properties of the illustrative AISI 52100 steel if it behaved in a completely ductile fashion--that is, if the crack could progress in fatigue across the entire failure section. The dotted curve is then obtained from the continuous curve by the procedure 
indicated in the previous section. It is seen that the data points on smooth specimens for this material, as shown by the circles, agree well with the dotted curve. Alternatively, if the experimental data for the smooth material is well established over the entire life range (that is, the dotted curve is well established by experiment), the curve for completely ductile behavior (continuous curve) can be obtained from the experimental curve by inverting the process described in the previous section, again using figure 12 to determine $N_{f}$ from the measured $\mathbb{N}_{f}^{\prime}$.

Having established the life characteristics of the smooth material on the basis of completely ductile behavior, its first use is to determine the number of cycles required to start a crack at the root of the notch. To accomplish this the strain range at the root of the machined notch is determined using the method described in reference 2 . Once the local strain at the root of the machined notch is known, the number of cycles at this strain level required to initiate the crack and cause it to grow to a size detectable with the unaided eye (assumed to be a depth of 0.003 inch from the root of the notch) can be determined from figures 8 and 9. At this point we start to take into account the 0.010 inch depth of the machined notch, and regard the total crack as extending to a depth of $0.013 \mathrm{inch}$, instead of 0.003 inch from the root of the notch. Concurrently, we revert to a consideration of crack growth associated with the nominal strain range in the test section instead of the local strain range at the root of the notch (ref. 2), and analytically determine how the crack would continue to grow as a function of cycles of load application if the material were completely ductile. From the value of $\mathrm{K}_{\mathrm{Ic}} / \sigma$, 
however, we can determine what the critical crack length is for brittle fracture. The number of cycles required to reach this critical crack length is the life of the notched specimen. This number of cycles can conveniently be determined with the aid of the auxiliary plot shown in figure 13(b). The use of this figure is discussed in appendix $C$. The calculated results are shown by the dot-dash curve of figure 13(a) accomparied by the experimental data points obtained on notched specimens. Very good agreement is seen to exist between calculations and experiment.

\section{Discussion}

Although good agreement has been found between the predictions of the simplified method outlined and the experimental data obtained for both the ductile and quasi-brittle materials evaluated, it must be emphasized that the number of tests is very limited and that considerably more data on other materials are needed before a conclusive evaluation of the validity of the method can be obtained. As more data are accumulated the following factors associated with the method may be reexamined:

(a) Consideration of the use of a normalized crack growth relation (eq. (5) and fig. 9) for all materials and for all life levels. Even if the assumption of such a relation is valid, its equation may be determined more accurately when more extensive data are available. It may also be observed that inherent in the use of a normalized crack growth relation is the assumption of the insensitivity of crack growth to notch toughness or other variables that do not directly manifest themselves in a determination of $\mathbb{N}_{f}$ for a given strain. Thus, two materials having the same values of ductility, tensile strength, and elastic modulus would be 
expected to have the same value of $\mathrm{N}_{f}$ at a given imposed strain range on a standard unnotched specimen (fig. I) in reversed strain cycling, regardless of the fact that the two may differ appreciably in notch toughness. They would thus have the same crack growth relation, and the same crack growth per cycle at any stage of crack growth length. This feature is inconsistent with other analyses of the problem of crack growth in fatigue. Krafft (ref. 10) has, for example, suggested that the crack growth per cycle is inversely proportional to $\mathrm{K}_{\mathrm{Ic}}^{2}$. Should this or some other assumption be a better representation of material behavior, it could be incorporated into the normalization of the crack growth relation.

(b) The form of the expression for $(\Delta \mathrm{V})_{f}$ should be reexamined as more data become available. It may be that the value of $P$ in the expression $\mathrm{PN}_{\mathrm{f}}^{0.6}$, or the exponent 0.6 , may be material constants. It may also be that a different expression for $N_{0}$ from that used in the analysis, especially in the low ranges of life, might be required. Perhaps of even greater interest is the expression of $N_{0}$ directly in terms of plastic strain rather than indirectly by first computing $\mathbb{N}_{f}$ and $(\Delta N)_{f^{*}}$ The relation between $\Delta \epsilon_{p}$ and $N_{O}$ would then be valid for geometric bodies other than the specimen of figure 1 , and would therefore have general utility. Likewise, instead of using a crack propagation period relation such as $14 \mathrm{~N}_{f}^{0.6}$, use could be made of more general expressions for crack growth in terms of plastic strain range per cycle, thereby again permitting the extension of the method to the treatment of more general geometries. The expression for $d l / d N$ in terms of $\Delta \epsilon_{p}$ discussed in appendix $B$ may serve as a starting point, but it must be refined 
when more data are available.

(c) The assumption of a normalized fracture relation (fig. 11) may require revision both in form and in the magnitude of the parameters involved in the expression for other materials.

(a) The problem of interpretation of computations of $\tau_{f}$ less than 0.013 inch requires investigation. Although a definite value of life does result from figure $13(b)$ for any value of $i_{f}$, it must be recognized that values which turn out to be less than 0.013 inch imply that brittle fracture occurs before the crack has penetrated a depth of 0.003 inch beyond the machined notch of 0.010 inch. Since the method of analysis makes use of relations developed on limited data obtained at crack depths greater than these values, it is evident that special attention would have to be given to cases for which $K_{I c} / \sigma<0.36 \sqrt{1 n}$. and for which $i_{f}<0.013$ inch.

Despite the foregoing limitations, some of which might readily be overcome by changes in the quantitative relationships, the method is of interest because of its simplicity and because it quickly yields definite answers in cases where attempts at more exact analyses might greatly complicate the process. It may therefore be applied with some confidence, since it yields good results for the case illustrated, but not without a recognition of its as-yet speculative features and potential limitations. INTERFACE BETWEEN FATIGUE AND CREEP

We consider now the interface between fatigue and creep represented by the problem of fatigue at high temperatures. It has long been recognized that at absolute temperatures exceeding about half the melting point 
materials are susceptible to time-dependent deformation under steady load, this type of deformation being known as creep. One of the features of creep failure is the large amount of associated intercrystalline cracking, in contrast to transcrystalline failure often characteristic at temperatures below the creep range. The question arises, therefore, as to the importance of such intercrystalline cracks on the fatigue behavior of a material at temperatures within the creep range.

In approaching this problem a natural question arises: How accurately would we predict the fatigue behavior at the high temperature if we proceeded in the same manner as at room temperature, for example by applying the method of universal slopes? Obviously we would expect to use the tensile properties measured at the temperature of interest, but is this enough? In the creep range the properties are very sensitive to strain rate, so that we might expect tensile strength and ductility to depend on the rate at which we apply the strain in the tensile test. Thus, at the very least, we would expect that some time-factor would have to be introduced into the process of obtaining the tensile properties and applying them to the analysis.

Attempts to predict high temperature fatigue behavior by using properties obtained in a tensile test at the temperature of interest result, in general, in predictions of longer lives than are obtained by fatigue experiments. In part the discrepancies may be due to the differences in strain rate imposed in a tensile test and in a fatigue test. Coffin (ref. 11), for example, discusses this factor in connection with a material 
which is strongly strain-aging. However, not all unconservative predictions can be so explained. For example, tests were conducted in the author's laboratory at nominal strain rates which were approximately equal to the strain rate in the conventional tensile test. These tests still resulted in larger discrepancies between predicted and experimental fatigue properties than would be expected on the basis of experience with numerous materials at room temperature.

\section{Intercrystalline Cracking Hypothesis}

In order to explain at least part of the discrepancies, we resort to a consideration of the importance of the phenomenon often characteristically associated with the creep range, namely intercrystalline cracking. That such cracking is common in fatigue in the creep range is illustrated in figure 14 for a cobalt-base alloy L-605 and a high-temperature steel A-286. Numerous other investigators have found such cracking common in other materials as well. Wood and Nine (ref. 12) have recently studied copper at room temperature and at $400^{\circ} \mathrm{C}$, and have found intercrystalline cracking characteristic of the $400^{\circ} \mathrm{C}$ tests but essentially absent in the room temperature tests.

The importance of intercrystalline cracking in fatigue can best be emphasized by considering the relative importance of crack initiation and propagation in fatigue, especially in the intermediate cyclic life range. As an illustration, consider a specimen which would survive for $10^{4}$ cycles at a temperature below the creep range (fig. 15(a)). In the early cycles of loading the straining causes cyclic slip along favorably-oriented slip planes of selected individual crystals. Figure 15(a) shows schematically 
cracks that have nucleated in the slip planes of individual crystals, and have linked together to form a crack of more appreciable dimension. The mechanism of early crack formation has been studied by Forsythe (ref. 13) and Wood et al. (ref. 14) among other investigators. Initially the crack may be confined to individual slip planes (or closely spaced planes forming bands) of individual grains, and the cracks may grow within these confined bands until they reach the crystal boundaries. Eventually these microcracks link and form a macrocrack. In figure 15(a) the macrocrack is shown as approximately 3 to 4 grains deep, which for a material with an average grain dimension of 0.001 inch, implies a crack depth of approximately 0.003 inch. The number of cycles required to generate a crack of this depth at a strain range for which $N_{f}=10^{4}$ can be estimated from figure 9. According to this figure, when $l=0.003$ inch the remaining Iife of an unnotched specimen is $6.2 \mathrm{~N}_{\mathrm{f}}^{0.6}$ for $\mathrm{N}_{f}>730$ cycles. Therefore, for $\mathrm{N}_{f}=10^{4},(\Delta \mathrm{N})_{f}=6.2 \mathrm{~N}_{f}^{0.6}=0.15 \times 10^{4}$, making $\mathrm{N}_{0}=0.85 \times 10^{4}$. Thus, in this case, the number of cycles required to initiate a crack and to propagate it to a depth of approximately 3 grains deep is 85 percent of the total life to fracture.

If we now assume that intercrystalline cracking serves the same purpose in generating the initial crack as do the slip plane cracks in the case illustrated in figure $15(a)$ we get the result shown schematically in figure 15(b). We must first decide how many cycles are required to cause an intercrystalline crack to progress 3 to 4 grain depths. It can be assumed that in the creep range intercrystalline cracks form much more readily than slip plane cracks. We consider, therefore, the extreme case 
in which such intercrystalline cracking occurs immediately upon the application of the first cycle of loading. If this were actually the case, then 85 percent of the life in the sub-creep range, which was the period required to generate the 0.003 inch crack, would now be bypassed. Next we must decide how much of the 15 percent of the life associated with propagating the crack from a length of 0.003 inch to complete failure remains in the elevated temperature test. Some intercrystalline failure is characteristic of the crack propagation as well as crack initiation. However, if we assume, in the extreme, that none of the crack propagation period is reduced as a result of intercrystalline cracking, the remaining life is still only 15 percent of the computed value for the sub-creep range. From the above simple example it is evident that one way of considering the significance of intercrystalline cracking associated with the creep range is to assume that such intercrystalline cracking replaces the process of crack formation by slip-plane plasticity which normally requires a large number of cycles. Below the creep range the grain bourdaries act as impediments to crack propagation because of the need for changing direction of slip planes in adjacent grains (fig. 15(a)), but in the creep range the grain boundaries contribute directly to the formation of cracks. The reason for this grain boundary cracking may be associated with the high degree of atomic disorientation and concentration of dislocations in the grain boundary region. Self-diffusion, a mechanism contributing to creep deformation (ref. 15) may thus be enhanced in these boundaries. As a result, the imposed strain tends to be concentrated in the grain boundary regions rather than being more uniformly distributed 
throughout the bulk of the material, and intercrystalling cracking occurs. While the foregoing discussion suggests a possible approach to the study of high-temperature creep, the quantitative application of the approach must take into account the actual reduction in both the crack initiation phase and the crack propagation phase. The reductions may differ from material to material, being dependent on the number of grain boundaries present and their properties, and, of course, they will depend on temperature and strain rate. Data are not yet adequate to undertake an accurate quantitative analysis, but we may use such data as are available to point to expected trends. To this end we have examined the high temperature fatigue data from various sources (refs. 16 to 19), comparing the experimental results to predictions that were made according to the method of universal slopes.

Correlation With Experiment

In figure 16 we have plotted as data points the ratios of observed fatigue life to the predicted lives based on tensile properties at the same temperature as the fatigue tests. It is evident that the calculated lives are considerably higher than the actual lives, since the plotted values fall well below an ordinate value of unity which represents exact agreement.

Also included in figure 16 are several life prediction curves based upon different assumptions regarding the crack initiation and propagation periods. For example, the uppermost curve represents the assumption that no crack initiation stage is required to produce a crack of about 0.003 inch in depth; but that the complete crack propagation stage is preserved. 
As shown in figure 9, this propagation stage is equal to $0.443(\triangle \mathrm{N})_{f}$ cycles, in which $\mathrm{N}_{f}=(\Delta \mathrm{N})_{\mathrm{f}}$ for lives below 730 cycles. Otherwise, $\left(\Delta V_{f}\right)_{f}$ is equal to $14 N_{f}^{0.6}$ cycles. It is seen from the uppermost curve, therefore, that the percentage of life which remains at elevated temperatures is constant up to a predicted life of 730 cycles, and progressively decreases for higher computed lives.

In a similar manner curves associated with other assumptions are shown in figure 16. The 5 percent-50 percent rule, for example, implies a 5 percent retention of the 0.003 crack initiation phase and a 50 percent retention of the phase required to propagate the 0.003 inch crack to failure. The solid line is based on the extremely simple assumption that 10 percent of both crack initiation and propagation phases are retained. In this case it is not even necessary to separate the two phases, since the total life is now also only 10 percent of the predicted value.

It is clear from figure 16 that no single simple rule agrees with all the data points shown. There does seem to be a tendency for the data points to be lower the longer the life of the specimen, thus implying that the crack initiation period is more severely affected than the crack propagation period. This trend is reflected in the prediction curves, except of course, for the 10 percent rule.

Some benefit might result from seeking a rule that is in best agreement with the experimental data, for example the 8 percent-25 percent rule shown by the short dash curve. However, more accurate data on many materials will be required before it can be determined whether such a rule can emerge. Of special interest, however, is the 10 percent rule. Its great 
simplicity, not requiring even the separation of the life into two phases, makes it ideal for application as a first-approximation rule of thumb. It will, therefore, be included in all of the detailed analyses to follow.

Figure 17 shows the more detailed analysis of the data of Gohn and Ellis (ref. 20) for lead at room temperature (which is within its creep range because of its low melting point). These investigators did not publish static tensile properties for the several types of lead studied, so that it is not possible to make the analytical predictions of hypothetical life according to the method of universal slopes. Fortunately, however, tests were conducted at both the high frequency of $1650 \mathrm{cpm}$ and the Low frequency of $1 / 4 \mathrm{cpm}$. Since the time available for creep effects is much less at the high frequency of straining than at the low frequency, we can resort to an alternate approximation. We shall assume that the data for the high frequency tests represent the results that would have been obtained by computation from the tensile properties, and use these data to compute lives for the low frequency tests by several of the rules previously cited. These high frequency tests are shown in figure 17 by the circles and dashed curves. Computations based on the 10 percent rule are shown by the continuous curves, and those obtained by the 5 percent-50 percent rule are shown by the dot-dash curves. For the pure lead as well as the chemical and antimony grades it is seen that reasonable agreement exists between the experimental data at $1 / 4 \mathrm{cpm}$ and the predictions of the two rules using the $1650 \mathrm{cpm}$ tests as the basis for the computations. There is a definite trend for the data to indicate a greater percentage reduction at the higher lives than at the lower lives, implying that the 
crack initiation phase is reduced more by the intergranular cracking than the crack propagation phase. That intercrystalline cracking was characteristic of these tests was observed by Gohn and Ellis.

Of special interest are the tests on calcium lead. At room temperature little intercrystalline cracking was observed, the computations overcorrect, and predict considerably lower lives than were actually obtained. When the test temperature was raised to $180^{\circ} \mathrm{F}$, however, intercrystalline cracking appeared more notably, and the data are in better agreement with the analytical predictions. The corrections appear, therefore, to be most important when intercrystalline cracking is present.

A more complete analysis of the $\mathrm{Cr}-\mathrm{Mo}-\mathrm{V}$ steel data at room temperature and at $1050^{\circ}$ F obtained by Coles and Skinner (ref. 17) is shown in figure 18. In figure 18(a) are shown the experimental data and the analytical predictions at room temperature. The dashed curve in this figure represents the predictions for the low-cycle fatigue behavior determined from the method of universal slopes (fig. 3) using the static tensile properties obtained by Coles and Skinner. Good agreement is seen to exist at room temperature where creep is negligible in this material. When the $1050^{\circ} \mathrm{F}$ static tensile properties were used to predict the fatigue properties, the predictions are again higher than the experimental data as shown in figure 18(b) by a comparison between the dashed curve and the experimental data points. The experimental points are shown for a large range of frequencies, and it is apparent that the tendency is toward lower fatigue life as the strain rate is lowered. However, the effect of frequency 
is not as large as might be expected for the wide range of frequencies involved. This relative insensitivity to very low frequencies may be due in part to the fact that they were obtained by introducing hold-periods at maximum strain, which would introduce some relaxation of the stress. The corrected predictions based on the 10 percent rule and the 5 percent-50 percent rule are shown by the solid and dot-dash curves respectively. The 10 percent rule apparently overcorrects over the entire life range while the 5 percent-50 percent rule agrees well with the data at the lower cyclic lives, and overcorrects only in the higher range of lives tested. However, the only data given in the higher life range are for the highest frequency used in the tests, where the creep corrections would be expected to be the lowest. Even where overcorrection occurs, the predictions by both rules represent reasonable approximations of the experimental data considering that no fatigue tests whatsoever are used to make the predictions.

The results for the data by Forrest and Armstrong on Nimonic 90 (ref. 16) are shown in figure 19. Again, the room temperature predictions by the method of universal slopes, based on the tensile properties cited by the authors, are shown in figure 19(a) by the dashed curve. Very good agreement with the experimental data exists. The dashed curves in figures $19(b)$ to (d) are the predictions by the method of universal slopes at temperatures respectively of $1380^{\circ}, 1600^{\circ}$, and $1800^{\circ} \mathrm{F}$, using the static tensile properties at these temperatures to make the predictions. In all cases these dashed curves overestimate the lives at all strair 
levels compared with those obtained experimentally. Again it may be noted that in general, the experimental data at the lower frequency of 0.1 cpm yields lower life values at a given strain range than the higher frequency $10 \mathrm{cpm}$ tests. The difference becomes less pronouned as the temperature is increased, and the creep effect becomes important for both frequencies. Reasonably good agreement is seen between the predictions of both the 10 percent rule and the 5 percent-50 percent rule at $1600^{\circ}$ and $1800^{\circ} \mathrm{F}$. At $1380^{\circ} \mathrm{F}$, however, considerable overcorrection occurs by both rules in the higher cyclic life range. It would be expected that the discreparcy would be the greatest at the lowest temperature where the creep effects are the lowest.

\section{Discussion}

Examining the results shown in figures 16 to 19 would lead to the conclusion that even if the explanation speculatively offered for the hightemperature effect is not rigorously correct, the procedure for estimating high temperature fatigue lives arising out of this explanation produces reasonably correct results, considering that no fatigue tests at all are required for obtaining these estimates. It is, of course, not yet clear what the relative effect of intercrystalline cracking is on the initiation and propagation periods, and tests designed to clarify this point would be very valuable. The $1 C$ percent rule, which attributes equal losses to both phases, possibly undercorrects the crack initiation phase and overcorrects the crack propagation phase. These opposite effects may balance each other, and produce reasonable results in a limited life range for small specimens.

Since the intent of the correction is to account for intergranular 
cracking it will obviously result in an overcorrection, when such cracking does not occur. This was already shown as a possibility in connection with calcium lead (fig. 17(b)). Perhaps of even greater interest is the hypothesis that the avoidance of intercrystalline cracking results in the achievement of a greater fraction of the fatigue strength potential of a material in the high temperature range--assuming that the computations based on the tensile properties are some measure of the potential fatigue strength of the material. This would imply that material development for applications involving high temperature fatigue should include the search for mechanisms of strengthening, protecting, or even removing some of the grair boundaries.

CUMULATIVE FATIGUE DAMAGE CONSIDERED AS A PROCESS OF CRACK

\section{INITIATION AND PROPAGATION}

As a final subject we shall now consider the question of cumulative fatigue damage from the standpoint of the concepts of crack initiation and propagation already outlined. It is well known that the linear damage rule for a spectrum of loading, which indicates that a summation of cycle ratios is equal to unity, is not completely aceurate. By spectrum-type loading is meart the successive application of load at different stress levels. The linear damage rule is also commonly referred to as the Miner hypothesis and is based on work done first by Palmgren (ref. 2l) and later by Langer (ref. 22) and by Miner (ref. 23). It is almost always used in design because of its simplicity and because it has been found to be in reasonable agreement with experimental data for certain cases. If a rew method is to replace the linear damage rule in practical design it is 
important that this method retain much of the simplicity of the linear damage rule.

In order to approach the question of an alternate method we must examine first some of the reasons for the shortcomings of the linear damage rule. One deficiency is that it does not take into account the effect of order of loading. For example, it has been found experimentally that in a two-step fatigue test high load followed by low load produces a cycle summation less than unity, while low load followed by high load produces a cycle summation greater than unity. A second deficiency is that it does not properly account for the effect of residual stress, especially during the crack propagation stage. If the period of high load cycling is ended after the application of a tensile stress, residual compressive stresses remain at the root of the notch; in the subsequent application of lower load numerous stress cycles, or perhaps a rest period (ref. 24), may be required to overcome this residual stress field before the crack will start propagating again. Another limitation is that it does not take into account stress cycles below theinitial fatigue limit of the material if such a fatigue limit exists. Cycle ratios of stresses below the initial fatigue limit are always zero when the linear damage rule is applied, whereas in reality the effect of prior loading may reduce the fatigue limit, implying that cycle ratios of stresses below the initial fatiglie limit should perhaps be accounted for (ref. 25). A further limitation is that the linear damage rule does not take into account "coaxing" effects present in some strain-aging materials (ref. 26) in which the appropriate sequence of loading may progressively raise the fatigue limit. Other 
synergistic effects, whereby sequential louling produces results not present when the individual loads act alone are also neglected in the application of the usual linear damage rule.

A number of methods have been proposed as alternatives to the linear damage rule. None overcomes all of the deficiencies. In the following discussion we shall consider the merit of treating the crack initiation and propagation phases separately, and applying a linear damage rule to each. The principal effect will be found in correcting deficiencies associated with order of loading; the other limitations cited above still will not be taken into account by this new approach.

The concept of improving cumulative fatigue damage predictions by separation of the crack initiation ard propagation phases was first suggested by Grover (ref. 27), but no rational basis for this approach was indicated, nor were definite expressions provided for separating out the two phases. These two aspects will now be considered in greater detail. Ore of the features characteristic of a strain cycling fatigue test is the progressive change of load required during the early portion of the test in order to maintain a fixed strain range. Basically the hardening or softening associated with the early phases of the test is believed to be a manifestation of rearrangement of dislocations or cell structure within the grains of the material (ref. 1, fig. 8). If, instead of maintaining a single strain level until failure occurs, changes in strain level are introduced during the course of the test, it is found that hardening or softening takes place in such a manner as to be predictable by a linear damage rule from the strain hardening or softening curves obtained in 
fatigue tests at a single strain level (e.g., ref. 1, fig. 43). Thus, if we assume that crack initiation is governed to some extent by the same microstructural changes as influence the hardening and softening characteristics, we may have the beginnings of a rationale for assuming a linear damage rule for crack initiation.

As for the validity of a linear damage rule in the crack propagation phase we refer again to reference $1(p .215)$ where the question of crack growth was discussed. It was demonstrated (ref. 1, fig. 46) that if a single curve resulted when crack length was plotted as a percentage of fatigue life for twu more strain levels, then a linear damage rule would be valid. The discussion there related to total life, but the concept could equally be applied if life is measured from the point of initiation of the crack, rather than total life. That a plot of crack length versus fraction of crack propagation period results in a single relation for all strain ranges is implied by equation (4), if $l_{f}$ and $l_{0}$ are assumed to be constants as already discussed. Thus we have in equation (4) a tentative rational basis for the assumption of a linear damage rule for the crack propagation phase.

\section{Two Cumulative Fatigue Damage Rules}

The first problem in the treatment of cumulative fatigue, therefore, is to establish expressions for the effective crack initiation and crack propagation phases. At this early state of development of the theory there is a certain degree of arbitrariness associated with the functional forms of the relationships to be assumed for the crack initiation and propagation stages. However, being guided by the desire to maintain a consistercy with 
the form of the equation for plastic strain in terms of life associated with the method of universal slopes, we shall assume that the crack propagation period $(\Delta N)_{f}$ can be written in terms of the life $N_{f}$ by the equation

$$
(\Delta \mathrm{N})_{\mathrm{f}}=\mathrm{PN}_{\mathrm{f}}^{\mathrm{O}} \cdot 6
$$

where $\mathrm{P}$ is an adjustable constant later to be determined so as to be consistent with a limited amount of experimental data available. For the present we shall avoid the complication of considering the inclusion of tests for which $\mathrm{PN}_{f}^{0.6}>\mathrm{N}_{f}$, so that the crack propagation period expressed by equation (9) is always less than the total life. For this case the crack initiation period is

$$
N_{O}=N_{f}-P N_{f}^{O .6}
$$

Thus the two equations of the double-linear damage rule become for

$$
\sum \frac{n}{N_{0}}=\sum \frac{n}{N_{f}-P N_{f}^{0.6}}=1
$$

for initiation, and

$$
\sum \frac{\mathrm{n}}{(\Delta \mathrm{v})_{\mathrm{f}}}=\sum \frac{\mathrm{n}}{\mathrm{PN}_{\mathrm{f}}^{0.6}}=1
$$

for propagation.

A convenient method for determining the value for $P$ is to conduct a test at a high load range for a part of the life, then continuing the test to failure at a lower load range. Specifically, if we conduct a test using the strain at which the life would be 1000 cycles as the initial level, and a strain at which the life would be 500,000 cycles as the level for completion of the test, the expected results would be 
as shown in figure 20. For a value of $P=14$ the predicted behavior would be the line ABG; if $P$ were 12 the line ACG would be the behavior, etc. If a linear damage rule applied for the total life values as predicted by Palmgren-Miner, the behavior would be shown by the dashed line AG. Experimental data for a maraging steel are shown on the figure by the circles, implying that a value of $P=14$ correlates the experimental behavior better than any of the other values shown for this material. The steepness of the portion $A B$ is associated with the fact that, for a value of $P=14$ the first 100 cycles of $n_{1}$ are sufficient to start the crack; once the crack is formed the remaining life at the strain level for which the life would have been 500,000 is greatly reduced, since the crack initiation period for this strain range would have been a much greater fraction of the 500,000 cycle life, and this crack initiation period is now bypassed by only a small life-fraction ratio at the higher strain level.

It is recognized, of course, that figure 20 represents only very limited data on only one material for the determination of $P$, and limited evidence as to whether the entire concept is indeed correct. However, it is interesting to proceed on the basis that it is correct and to determine the implications of this assumption by examining other data that have been published.

Comparison With Previously Proposed Cumulative Damage Theory In 1961 we examined some of the deficiencies of the Palmgren-Miner Iinear damage rule, and proposed a method for correlating cumulative fatigue data (ref. 28); this approach was extended in a subsequent paper 
(ref. 25). It is instructive, therefore, to re-examine the method and the data of these two papers in the light of the double linear damage hypothesis suggested by equations (11) and (12).

The basis for the method proposed in reference 28 lay in the observation of the $\mathrm{S}-\mathrm{N}$ fatigue curves obtained on specimens that were precycled short of failure at a different stress level. Schematically, the results obtained were as shown in figure $2 I(a)$. The Iine QA is the S-N curve of the original material. Precycling resulted in $S-N$ curves $Q B$, QC, etc. having the general appearance of convergence with QA at a common point $Q$. The larger the cycle ratio of the pre-stress the more the rotation of the remaining $S-N$ curve. On the basis of the Palmgren-Miner linear damage rule the remaining $S-N$ curves would have been a family of lines parallel to the initial S-N line QA. The difference between the convergence and the parallel nature of the remaining $S-N$ curves can be used to explain the effect of order of load application (ref. 25). In a two-step fatigue test it can be shown that convergence tends to result in a predicted cycle ratio summation greater than unity if the low stress precedes the high stress, and a summation less than unity if the high stress is applied first.

The corresponding implications of the double linear damage rule are schematically shown in figure $2 I(b)$. Obtaining the lines $Q A, Q B$, etc., which represent the remaining $S-N$ curves after precycling at an arbitrary stress level, is a relatively simple matter. The value of $\mathbb{N}_{0}$ is first determined for the prestress condition using equation (10) and the corresponding value of $\mathbb{N}_{f}$. The cycle ratio for the prestress condition is then determined in relation to crack initiation by dividing $n_{1}$, the number 
of cycles applied, by $\mathrm{N}_{O}$. If this ratio is less than unity, then the crack initiation process continues at the second stress level, the number of cycles required to initiate the crack being determined by equation (II). Once the crack has started, the number of cycles at the second stress level required to cause failure is given by equation (12). Thus, the number of cycles that can be sustained at the second stress level is the sum of the cycles required to continue the process to crack initiation and those required to propagate the crack to failure. Those cases in which the prestress cycle ratio is not sufficient to cause crack initiation are represented by lines such as $Q B$ and $Q C$ which have the general appearance of convergence with the initial S-N line $Q A$. The point of convergence $Q$ is not strictly a common point for all prestress conditions. For this type of sequential loading the double linear damage rule is consistent with the method of reference 28 .

If, however, the prestress condition results in a crack initiation cycle ratio, $n_{1} / N_{0}$, greater than unity, then the implication is that the crack initiation process has been completed in the prestress portion, and that some of the crack propagation process has already been started in this portion as well. Equation (10) gives the number of cycles required to initiate the crack, and the remaining number of cycles in the prestress condition are then entered into equation (I2) as a cycle ratio for crack propagation. The number of cycles at the second stress condition to complete the crack propagation is then determined by equation (12). When a plot is made of the number of cycles at the second stress level that can be sustained before failure, Iines such as $\mathrm{RD}$ and $\mathrm{SE}$ are obtained 
parallel to $Q C$ when the pre-stress cycles were sufficient to at least start the crack. The Iine $\mathrm{QC}$ is the locus of points at which the pre-stress is just sufficient to initiate a crack, that is QC is obtained from QA by application of equation (10). Thus the double linear damage rule implies that prestressing does not continue to rotate the remaining $\mathrm{S}-\mathrm{N}$ curve indefinitely as the prestress cycle ratio is progressively increased; rather there is a limit to the amount of rotation that will be imposed, and beyond this limit further increases of prestress cycle ratio simply displace the remaining $\mathrm{S}-\mathrm{N}$ curve parallel to itself. This geometrical implication is more satisfying than the indefinite rotation implied by the method of reference 28. Although not of practical significance because it would require the most extreme of conditions, the method of reference 28 could require the remaining S-N curve to become vertical, and even of positive slope, which would of course be completely meaningless. The double-linear damage rule points to a way around this hypothetical difficulty .

\section{Correlation With Experiment}

A more complete analysis of the data previously obtained in our laboratory for verifying the method of references 28 and 25 is shown in figure 22. In these tests several materials were prestressed in rotating bending at one stress level for a number of cycles which did not cause failure, and the S-N curve of the prestressed material was subsequently obtained by conducting ordinary rotating bending tests at a number of stress levels. The particular tests conducted are identified in figure 22 . The dashed lines in each case represent the predictions by the double 
linear damage rule, using the procedure outlined. Reasonably good agreement between the predictions and experimental data is observed. Note especially in figure 22(a) that the data represented by the diamond symbols are beginning to show a parallel displacement of the S-N curve, as predicted by the double linear damage rule, rather than progressive rotation.

It is of interest to determine the range that might be expected in the cycle ratio summation based on total lives (the Palmgren-Miner procedure) if we assume the correctness of the double linear damage rule. Figure 20 has already shown that this summation can be considerably less than unity if the prior cycling is at the higher stress level. At point B, for example, the prestress is only maintained for approximately 10 percent of the life, while the number of cycles sustained at the second stress level is also only 10 percent of its life. Thus the combined cycle ratio based on total life is only approximately 0.2 for this case when the high stress was applied first. The differences between the predictions of the double linear damage rule and the Palmgren-Miner hypothesis become most pronounced as the two stress levels are separated farther and farther from each other (so that the relative proportions of periods spent in the crack initiation and propagation stages are as far different as possible between the two stages), and as the number of cycles in the first step more closely approaches the point of crack initiation. The deviations between a Palmgren-Miner linear damage rule and experiment will depend thus on the choice of test variables, and either a large discrepancy or a small one can be arranged if the test conditions are chosen judiciously. Based on double linear damage rule computations, cycle ratio summations 
between 0.2 and 1.8 can be shown to result for two-step fatigue tests if the steps are at loadings corresponding to total lives of $10^{3}$ and $10^{6}$ cycles. The low summation applies when the high stress is applied first; the high summation applies when the lower stress is applied first. The majority of experimental data from investigations that have been reported fall within the extremes of cycle ratio summation.

\section{Discussion}

Although the double linear damage hypothesis does appear to have potential, being extremely simple and valid in the limited cases examined, it must be pointed out that in general it overcomes only one shortcoming of the Palmgren-Miner linear damage rule, namely the effect of the order in which high and low stresses are applied. Residual stress, progressive charges of fatigue limit, coaxing, and other synergistic effects are no more accounted for by this approach than by the simple linear damage rule. Progressive change in fatigue limit could be included in this method, using the same concepts described in reference 28 . The other limitations still require attention. Also requiring attention is the case where one of the stress levels would have resulted in a life less than 730 cycles (or when $\mathrm{N}_{f}-\mathrm{PN}_{f}^{0.6}<0$ for other values of $\mathrm{P}$ ). A method could be developed using an approach similar to that described in connection with the fatigue failure of quasi-brittle materials, establishing a hypothesis for the crack growth for the low cyclic lives of $\mathrm{N}_{f}$ instead of $\mathrm{PN}_{f}^{0.6}$. However, because of the very limited data in this range of the variables available for checking the validity of the approach, the details will be omitted here. Of special interest, however, is the fact that in a twostep test, if both steps are in a life range for which the crack initiation 
period is essentially zero, a Palmgren-Miner linear damage rule will apply. Such tests might act as critical tests to establish the validity of the concept. In fact, a limited number of such tests (figs. 43-45, ref. 1) do appear to confirm this conclusion.

CONCLUDING REMARKS

The common thread in the treatment of the three problems discussed in this lecture is the value of separating the fatigue process into one of crack initiation and crack propagation. That such a separation is desirable is now becoming more and more recognized in other published investigations. The difficulty is in defining the meaning of a crack and assigning quantitative formulae to use in computations involving these phases. A first approach only is taken in this report toward this quantitative aspect.

While the application is intended for the analysis of laboratory specimens rather than engineering structures, the principles involved may perhaps be extended to practical geometries. For example, we have used local strain as a measure of crack initiation, and therefore the relations established between strain range and cycles to crack initiation may continue to be valid for other geometrles as well as the test specimen from which the relations are involved. The crack growth process would, however, depend on geometry. Perhaps an approach such as that used in appendix B, expressing instantaneous crack growth in terms of microscopic local strain range, would prove more useful in the treatment of general problems, instead of attempting to express the crack propagation as a closed-form solution that would be applicable only to specific 
cases. Likewise the fracture condition for quasi-brittle materials, while treated specifically for the laboratory specimen, could be extended, according to the fast-developing field of fracture mechanics, to other geometries by the same method of treatment. Similar extensions could perhaps be made to the subject of cumulative fatigue, using small laboratory specimens to obtain information on crack initiation and crack growth laws together with fracture mechanics to obtain the damage rule for the crack propagation stage. Also, in the discussion of high temperature the main emphasis has been on intercrystalline cracking. But the principles involved may have more significant generality. A similar approach may have utility in the treatment of surface imperfections and pernicious environments, that would also have the effect of cutting short the crack initiation period more than the propagation period.

In conclusion, it is important to emphasize again that the discussion is intended to suggest approaches rather than to present proven methods. Verification of the methods as presented, or indicated directions for improved approaches, must await further analyses and experiments.

\section{ACKNOWLEDGEMENT}

The author is grateful to the following of his colleagues for conducting experiments and assisting in the analysis of the various subjects covered in this lecture: M. H. Hirschberg, for the treatment of notch effects in quasi-brittle materials; D. A. Spera, for the high-temperature fatigue studies; and C. R. Ensign and J. C. Freche for the studies of cumulative damage in fatigue. 
APPENDIX A - APPLICATION OF METHOD OF UNIVERSAI SLOPES TO PREDICTION OF CYCIIC HARDENING OR SOFTENING CHARACTERISTICS

A material is said to experience cyclic strain hardening, neutral hardening, or cyclic strain softening if a positive, zero, or negative change, respectively, is observed in the stress range during a straincycling test. In a fatigue test, the elastic and plastic components of the strain range are usually recorded at their half-life values, when cyclic hardening or softening is essentially complete and "saturation" has been achieved. The change in stress range during cycling can therefore be estimated by comparing the actual "saturation" stress range predicted by the method of universal slopes with a neutral-hardening stress range calculated from the static stress-strain curve. This procedure is shown in figure 5(a). In this figure, the solid curve shows the relationship between the stress and strain ranges as predicted by the method of universal slopes and expressed parametrically in terms of the fatigue life $\mathbb{N}_{f}$. The dashed curve represents a neutral-hardening relationship, expressed parametrically in terms of the uniaxial strain $\epsilon$. For a strain range which is twice the uniaxial strain, the neutral-hardening stress range is twice the corresponding uniaxial stress. This follows from the fact that the stress-strain hysteresis loop has vertices at $(\epsilon, \sigma)$ and $(-\epsilon,-\sigma)$ for neutral hardening. If the estimated stress rangestrain range curve lies above the neutral hardening curve, the material experiences cyclic strain hardening; if it lies below the neutral hardening curve, the material undergoes cyclic strain softening.

Instead of considering changes in the entire stress range-strain range relationship, we can choose a single representative point for 
simplicity. For example, we may select the point of intersection of the lines in figure 3, point $\mathrm{T}$, which represents equal elastic and plastic components of the total strain range. The life at this intersection is designated as the "transition life" because it marks the change from plastic strain to elastic strain as the main component governing fatigue life. By the method of universal slopes, the value of the total strain range at the transition life is calculated to be

$$
\Delta \epsilon_{\mathrm{t}, T}=\frac{9.56}{\mathrm{D}^{0.15}}\left(\frac{\sigma_{\mathrm{u}}}{\mathrm{E}}\right)^{1.25}
$$

and the stress range is

$$
\Delta \sigma_{T}=E\left(\Delta \epsilon_{e l}\right)_{T}=\frac{4.78 \sigma_{u}^{1.25}}{D^{0.15} E^{0.25}}
$$

In figure 5(a), the neutral-hardening stress range corresponding to the transition strain range is designated as $\Delta_{O}, T$. A cyclic hardening coefficient may now be defined as

$$
C_{h}=\frac{\Delta \sigma_{T}-\Delta \sigma_{0, T}}{\Delta \sigma_{0, T}}
$$

If this factor is greater than zero, cyclic hardening is indicated; if it is less than zero, cyclic softening is predicted. Figure 5(b) shows the cyclic hardening coefficient for 30 materials. The experimental behavior for each material is indicated by the type of shading used in this figure. If the bar is shaded, the material actually experienced cyclic strain hardening during cycling. If no shading appears, softening was present. It can be seen that a reasonably good agreement was obtained 
with the predictions. Values of $C_{h}$ above zero almost always occurred for the strain hardening materials, while values of $C_{h}$ below zero almost always occurred for the strain softening materials. Thus, for this application also, the simple model of figure 3 for representing the strain cycling behavior in terms of the uniaxial static tensile properties yields reasonably good agreement with experiment.

$$
\text { APPENDIX B - FORM OF FUNCTIONS } f\left(\Delta \epsilon_{p}\right) \text { AND }(\Delta \mathrm{N})_{f}
$$

The form of the function $f\left(\Delta \epsilon_{p}\right)$ in equation (1), while not important to the derivation of equation (4), is nevertheless of general interest. Boettner, Laird and McEvily (ref. 29) and Weiss (ref. 30) have assumed $f\left(\Delta \epsilon_{p}\right)$ to be proportional to $\left(\Delta \epsilon_{p}\right)^{2}$. In reference $I$ we have also examined other power laws of $\Delta \epsilon_{\mathrm{p}}$. A reexamination of these assumptions has led us to question whether $f\left(\Delta \epsilon_{p}\right)$ can reasonably be taken as only a single term in $\Delta \epsilon_{\mathrm{p}}$ and still correlate available crack growth data in both the high and low life ranges. It would appear that a more suitable form might contain two terms, one that would predominate in the low life range, and the other in the high life range. Accordingly, one approach is to assume

$$
f\left(\Delta \epsilon_{p}\right)=A \Delta \epsilon_{p}+B\left(\Delta \epsilon_{p}\right)^{2}
$$

Substituting into equation (3) we would then get

$$
\ln \frac{l_{f}}{i_{0}}=\left[A\left(\Delta \epsilon_{p}\right)+B\left(\Delta \epsilon_{p}\right) 2\right]\left[N_{f}-N_{O}\right]
$$

In order to obtain a first approximation for the form of the term $\left[N_{f}-N_{Q}\right]$ we consider how equation (B2) degenerates for low values of $\Delta \epsilon_{p}$, such that $A\left(\Delta \epsilon_{p}\right)$ predominates over $B\left(\Delta \epsilon_{p}\right)^{2}$. Thus neglecting the 
$\left(\Delta \epsilon_{\mathrm{p}}\right)^{2}$ term, equation $(B 2)$ becomes

$$
\operatorname{In} \frac{l_{f}}{l_{O}}=A\left(\Delta \epsilon_{p}\right)\left[N_{f}-N_{O}\right]
$$

Equation (B3) and the universal-slope equation of plastic strain versus Iife (fig. 3) $\Delta \epsilon_{p}=D^{0.6}{ }_{N_{f}}^{-0.6}$ can be made to be consistent by assuming that

$$
N_{f}-N_{O}=P N_{f}^{O} \cdot 6
$$

The value of $P$ will tentatively be assumed as 14 consistent with the results of the cumulative fatigue tests as discussed in connection with equation (9) (recognizing that there may be some inconsistency involved in using rotating bending test data for analysis of axial fatigue tests). Since the value of $14 \mathrm{~N}_{\mathrm{f}}^{0.6}$ is greater than $\mathrm{N}_{f}$ for all values of $\mathrm{N}_{\mathrm{f}}$ less than approximately 730 cycles, it must be assumed that the crack growth period can only be designated by $14 \mathrm{~N}_{f}^{0.6}$ for values of $N_{f}>730$. Below $N_{f}=730$ cycles, the value of $N_{f}-N_{O}$ will be assumed to be equal to $\mathbb{N}_{f}$. The relation between $(\Delta \mathrm{N})_{f}$ and $N_{f}$, as well as the corresponding value of $N_{O}$ versus $N_{f}$, are shown in figure 8 .

Equation (B2) can now be used to determine the approximate values of $A$ and $B$. Substituting into equation (B2) for $\left(\mathbb{N}_{f}-\mathbb{N}_{0}\right)$ from equation ( $\mathrm{B} 4)$

$$
\text { In } \frac{\eta_{f}}{\tau_{0}}=\left[A\left(\Delta \epsilon_{p}\right)+B\left(\Delta \epsilon_{p}\right)^{2}\right] N_{f}
$$

for $N_{f}<730$ cycles, and

$$
\ln \frac{\tau_{f}}{\tau_{0}}=\left[A\left(\Delta \epsilon_{p}\right)+B\left(\Delta \epsilon_{p}\right)^{2}\right] 14 N_{f}^{0.6}
$$


for $\mathrm{N}_{f}>730$ cycles.

Since it is desirable to make equations (B5) and (B6) as consistent as possible with the universal relation $\Delta \epsilon_{p}=D^{0.6} \mathrm{~N}_{f}-0.6$ for all values of $\mathbb{N}_{f}$, a simple method of determining $A$ and $B$ is to select several values of $N_{f}$, determine $\Delta \epsilon_{p}=D^{0.6} N_{f}-0.6$, and substitute these values of $N_{f}$ and $\Delta \epsilon_{p}$ into equation (B5) or (B6), whichever is pertinent for the selected value of $N_{f}$. If more than two values of $N_{f}$ are chosen for application of the procedure a redundant set of equations results, the optimum solution for which can be found by least squares methods. Thus, applying the procedure to selected values of $\mathrm{N}_{f}$ in the range of $I$ to $10^{5}$ cycles and applying a least squares approximation gives:

$$
\begin{aligned}
& A=\frac{0.065 \ln \frac{l_{f}}{l_{O}}}{D^{0.6}}=\frac{0.065 \ln \frac{0.18}{1.8 \times 10^{-5}}}{D^{0.6}}=\frac{0.60}{D^{0.6}} \\
& B=\frac{1.150 \ln \frac{l_{f}}{l_{O}}}{D^{1.2}}=1.150 \ln \frac{0.18}{1.8 \times 10^{-5}}=\frac{10.6}{D^{1.2}}
\end{aligned}
$$

The crack growth law, equation (BI), becomes

$$
\frac{\mathrm{d} l}{\mathrm{dN}}=\left[\frac{0.60}{D^{0.6}} \Delta \epsilon_{\mathrm{p}}+\frac{10.6}{D^{1.2}}\left(\Delta \epsilon_{\mathrm{p}}\right)^{2}\right] \tau
$$

and equations (B5) and (B6) become respectively

$$
\mathbb{N}_{f}\left[0.065 \frac{\Delta \epsilon_{p}}{D^{0.6}}+1.150\left(\frac{\Delta \epsilon_{p}}{D^{0.6}}\right)^{2}\right]=1
$$

for $\mathrm{N}_{\mathrm{f}}<730$ cycles, and 


$$
14 N_{f}^{0.6}\left[0.065 \frac{\Delta \epsilon_{p}}{D^{0.6}}+1.150\left(\frac{\Delta \epsilon_{p}}{D^{0.6}}\right)^{2}\right]=1
$$

for $\mathrm{N}_{f}>730$ cycles. That equations (BIO) and (BII) represent essentially the plastic line according to the method of universal slopes can be seen in figure 23 where a plot is shown of $\Delta \epsilon_{p} / D^{0.6}$ versus $N_{f}$. The heavy solid line shows the power law relationship for plastic strain according to the method of universal slopes. The light curve and dotted section show the relation according to equations (BIO) and (BII) respectively. Little difference exists between the two types of representation of $\Delta \epsilon_{\mathrm{p}} / \mathrm{D}^{0.6}$ versus $\mathrm{N}_{\mathrm{f}}$. Thus it can be seen that the crack growth law equation (B9) can lead to the same representation of plastic strain against cyclic life as does the empirically determined method of universal slopes. While the consistency between the two forms of representation has been essentially forced by the choice of the constants $A$ and $B$, the agreement does serve to show that a power-law relation between plastic strain and life can be derived from crack growth considerations. It still remains to determine a crack growth equation such as (BI) from physical or geometrical considerations.

\section{APPENDIX C - IILUSTRATIVE CAICJIAATION FOR NOTCHED QUASI-BRITTLE SPECIMENS}

An illustrative example will be shown for an applied strain range of 0.00884 on a notched specimen of the material considered in figure 13 . For this applied strain and a nominal strain concentration factor of 2 , the method of reference 2 indicates an actual strain concentration factor of 2 , the method of reference 2 indicates an actual strain concentration 
factor of 2.23 for this material. Thus, the localized strain at the root of the notch is 0.01968 , for which $\mathrm{N}_{f}=758$ cycles according to figure 13 . At this value of $N_{f}$, the value of $(\Delta N)_{f}$ is $14(758)^{0.6}$ or 748 cycles. Thus $N_{0}=N_{f}-14 N_{f}^{0.6}=10$ cycles. This point is plotted at $A$ in figure $13(b)$. Since the period from $\mathbb{N}_{0}$ to a crack depth of 0.003 inch is $7.8 \mathrm{~N}_{\mathrm{f}}^{0.6}=415$ cycles by figure 9 , a crack depth of 0.003 inch beyond the root of the machined notch occurs at $(10+415)=425$ cycles. This point is plotted at $B$ in figure $13(b)$. At this depth of crack the procedure is to change abruptly from a crack depth of 0.003 to 0.013 inch (in order to take into account the $0.010 \mathrm{in.} \mathrm{machined} \mathrm{depth} \mathrm{of} \mathrm{the} \mathrm{notch),}$ and to change the applied strain level from 0.01968 back to the nominal strain range of 0.00884 . The starting point of the abrupt change is shown at $\mathrm{C}$ in figure $13(\mathrm{~b})$. The next step is to determine how the crack would progress across the entire section of the specimen if it were not interrupted by brittle fracture. According to figure 9 , the period of crack growth between 0.013 inch and 0.18 inch consumes $4.0 \mathrm{~N}_{f}^{0.6}$ cycles, where $\mathbb{N}_{f}$ now corresponds to the applied strain level of 0.00884 . Thus $D$ is plotted at $425+4 \mathrm{~N}_{\mathrm{f}}^{0.6}=425+4\left(10^{5}\right)^{0.6}=4425$ cycles at a crack length of 0.18 inch. However, for a strain range of 0.00884 corresponding to a stress amplitude of $129.3 \mathrm{ksl}$, the critical crack length for brittle fallure is $\left(K_{I c} / \sigma\right)^{2}=(17 / 129.3)^{2}=0.0173$ inch, since $K_{I c}=17 \mathrm{ksi}-\sqrt{i n}$. for this temper of material. Thus a horizontal line $E F$ may be arawn in figure $13(b)$ at 0.0173 inch. The intersection of this line with the crack growth Iine $C D$ is at $F$ where $N=860$ cycles. Thus the Iife 
dictated by quasi-brittle fracture is 860 cycies, which is plotted at point $F^{\prime}$ in figure $13(a)$ at an applied nominal strain range of 0.00884 . In this manner other points on the dot-dashed curve of figure 13(a) are determined for corresponding values of selected nominal strains. REFEREICES

1. Manson, S. S.: Fatigue: A Complex Subject - Some Simple Approximations. Experimental Mechanics, vol. 5, no. 7, July 1965, pp. 193-226. 2. Manson, S. S.; and Hirschberg, M. H.: Crack Initiation and Propagation in Notched Fatigue Specimens. Proposed NASA Technical Note.

3. Manson, S. S.: Behavior of Materials Under Conditions of Thermal Stress. Heat Transfer Symposium, University of Michigan, June 27-28, 1952 , University of Michigan Press, 1953, pp. 9-76. (Also available as Manson, S. S.: Behavior of Materials Under Conditions of Thermal Stress. NACA TNN-2933, 1953.)

4. Coffin, L. F., Jr.: A Study of the Effects of Cyclic Thermal Stresses on a Ductile Metal. Trans. ASME, vol. 76, Aug. 1954, pp. 931-950.

5. Smith, Robert W.; Hirschberg, Marvin H.; and Manson, S. S.: Fatigue Behavior of Materials Under Strain Cycling in Low and Intermediate Life Range. INASA TN D-1574, 1963.

6. Tavernelli, J. F.; and Coffin, I. F., Jr.: Experimental Support for Generalized Equation Predicting Low Cycle Fatigue. J. Basic Eng. vol. 84, no. 4, Dec. 1962 , pp. 533-537 (Discussion by S. S. Manson, pp. 537-541). 
7. Manson, S. S.; and Hirschberg, Marvin H.: Fatigue Behavior in Strain Cycling in the Low- and Intermediate-Cycle Range. Proceedings of the Tenth Sagamore Army Materials Research Conference, John J. Burke, Norman L. Reed and Volker Weiss, eds., Syracuse University Press, 1964, pp. 133-178.

8. Manson, S. S.: Materials Under Thermal Stress and Low Cycle Fatigue. Chapter 4, McGraw-Hill, to be published in 1966.

9. Srawley, John E.; and Brown, William F., Jr.: Fracture Toughness Testing Methods. Fracture Toughness Testing and Its Application, Spec. Tech. Publ. No. 381, ASTM, 1965, pp. 133-198.

10. Krafft, J. M.: On Prediction of Fatigue Crack Propagation Rate from Fracture Toughness and Plastic Flow Properties. Trans. ASM, vol. 58, no. 4, Dec. 1965, pp. 691-695.

11. Coffin, L. F., Jr.: Cyclic Strain and Fatigue Behavior of Metals in the Creep Range. Preprint DI-9, International Conference on Fracture (Sendai, Japan), Sept. 1965. Preprint vol. no. 3 (chap. C, $D$ and $E$ ), Organizing Committee of International Conference or Fracture, The Unified Research Society for Micro- and Macro mechanical Behavior of Materials, The Japan Society for the Promotion of Science, 1965.

12. Wood, W. A.; and Nine, H. D.: Differences in Fatigue Behavior of Single Copper Crystals and Polycrystalline Copper at Elevated Temperatures. Dept. of Civil Engineering and Engineering Mechanics, Columbia University, Feb. 1965. (Available from DDC as AD-612713.) 13. Forsyth, P. J. E.: Fatigue Damage and Crack Growth in Aluminum Alloys. Acta Met., vol. 11, no. 7, July 1963, pp. 703-715. 
14. Wood, W. A.; Cousland, S. McK; and Sargant, K. R.: Systematic Microstructural Changes Peculiar to Fatigue Deformation. Acta Met. vol. 11, no. 7, July 1963, pp. 643-652.

15. Dorn, John E.; and Shepard, Lawrence A.: What We Need to Know About Creep. Symposium Effect of Cyclic Heating and Stressing on Metals at Elevated Temperatures, Spec. Tech. Publ. No. 165, ASTM 1954, pp. 3-30.

16. Forrest, P. G.; and Armstrong, K. B.: The Thermal Fatigue Resistance of NickeI-Chromium Alloys. Joint Irtersational Conference on Creep. Aug. 25-29 and Sept. 30-0ct. 4, 1963, Inst. Mech. Eng. (London), 1963, pp. 3-1 to 3-7.

17. Coles, A.; and Skinner, J.: Assessment of Thermal-Fatigue Resistance of High Temperature Alioys. J. Roy. Aeron. Soc., vol. 69, no. 649, Jarı. 1965, pp。53-55.

13. Carden, A. E.: Thermal Fatigue of a Nickel-Base Alloy. J. Basic Erg., voI. 87, no. 1, Mar. 1965, pp. 237-244.

19. Coffin, I. F., Jr.: Cyclic Strain and Fatigue Study of A 0.1\% C-2.0\% Mo Steei. Rep. No. 64-RI-3612M, Gereral Electric Company, Mar. 1964. (Also available as Coffin, I. F'., Jr.: Cyclic Strain and Fatigue Stiday of A $0.1 \%$ C-2.0\% Mo Steel at Elevated Temperatures. AIME Met. Soc. Trans., vol. 230, no. 7, Des. 1964, pp. 1690-1699.

20. Gohn, G.R.; and ElIis, W. C.: Fatigue Test as Applied to Lead Cable Sheath. Proc. Am. Soc. Testing Mater., vol. 51, 1951, pp. 721-744. 21. Palmgren, A.: Die Lebensdauer von Kugellagern. ZVDI, vol. 68, no. 14, Apr. 1924, pp. 339-341. 
22. Langer, B. F.: Fatigue Failure from Stress Cycles of Varying Amplitudes. J. Appl. Mech., vol. 4, no. 4, Dec. 1937, pp. Al60-Al62.

23. Miner, Milton A.: Cumulative Damage in Fatigue. J. Appl. Mech., vol. 12, no. 3, Sept. I945, pp. Al59-Al64.

24. Weibull, Waloddi: Fatigue Testing and Analysis of Results. Pergamon Press, 1961, p. 132 .

25. Manson, S. S.; Nachtigal工, A. J.; Ensign, C. R.; and Freche, J. C.: Further Investigation of a Relation for Cumulative Fatigue Damage in Bending. J. Eng. Ind., vol. 87, no. 1, Feb. 1965, pp. 25-35. 26. Rally, F. C.; and Sinclair, G. M.: Influence of Strain Aging on the Shape of the S-N Diagram. Rep. No. 87, Dept. of Theoretical and Applied Mechanics, University of Iliinois, June 1955.

27. Grover, Horace J.: An Observation Concerning the Cycle Ratio in Cumulative Damage. Symposium on F'atigue of Aircraft Structures. Spec. Tech. Publ. no. 274, ASTM 1960, pp. 120-124.

28. Manson, S. S.; Nachtigall, A. J.; and Freche, J. C.: A Proposed New Relation for Cumulative Fatigue Damage in Bending. Proc. Am. Soc. Testing Mater., vol. 61, 1961, pp. 679-703.

29. Boettner, R. C.; Laird, C.; and MeEvily, A. J., Jr.: Crack Nucleation and Growth ir High Strain-Low Cycie Fatigue. Trans. AIME, vol. 233, no. 2, Feb. 1965, pp. 379-387.

30. Weiss, Volker: Analysis of Crack Propagation in Strain-Cycling Fatigue. Proceedings of the Tenth Sagamore Army Materials Research Conference, John J. Burke, Norman L. Reed and Volker Weiss, eds., Syracuse University Press, 1964, pp. 179-186. 


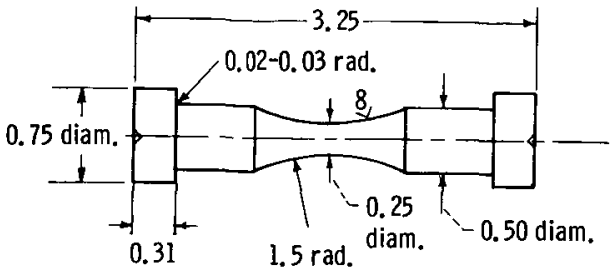

Figure 1. - Fatigue specimen configuration.

(Dimensions in inches.)
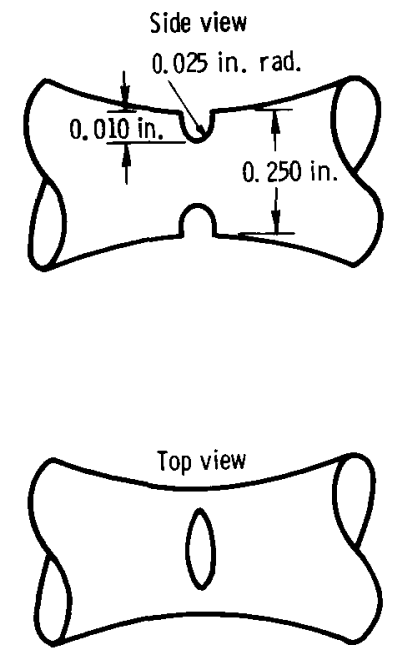

Figure 2. - Test section of notched fatigue specimen. 


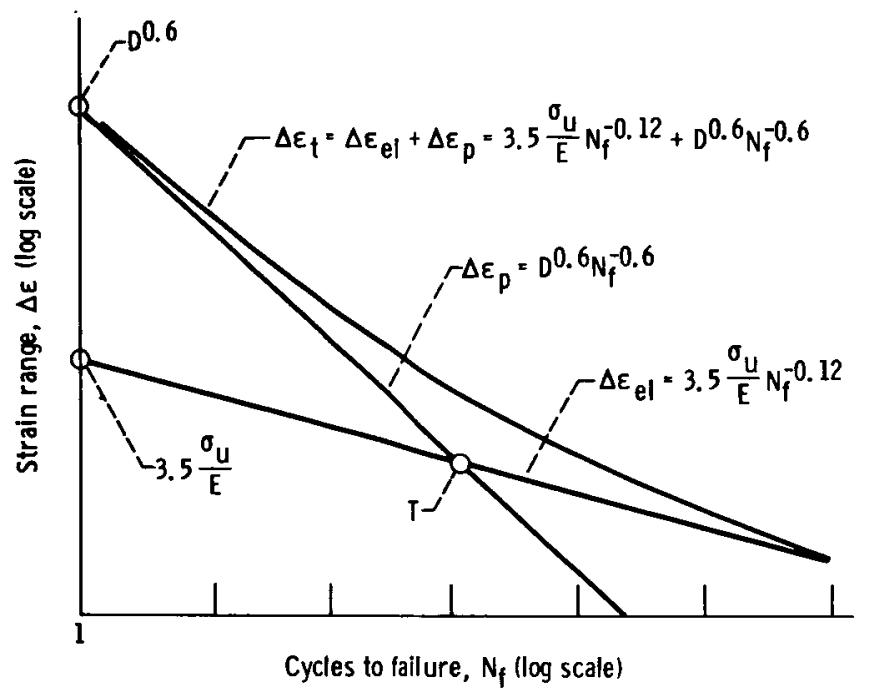

Figure 3. - Method of universal slopes for estimating axial fatigue life.

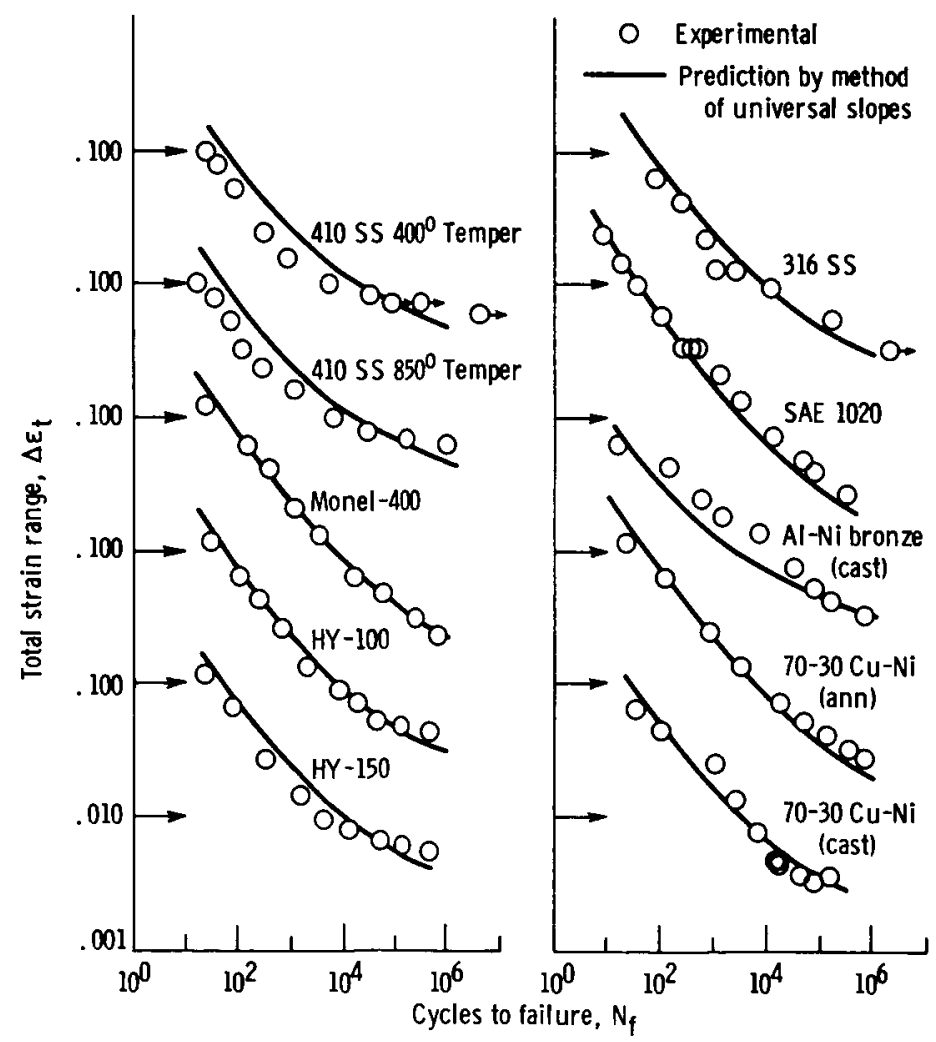

Figure 4. - Comparison of predicted and experimental fatigue behavior for several materials. 


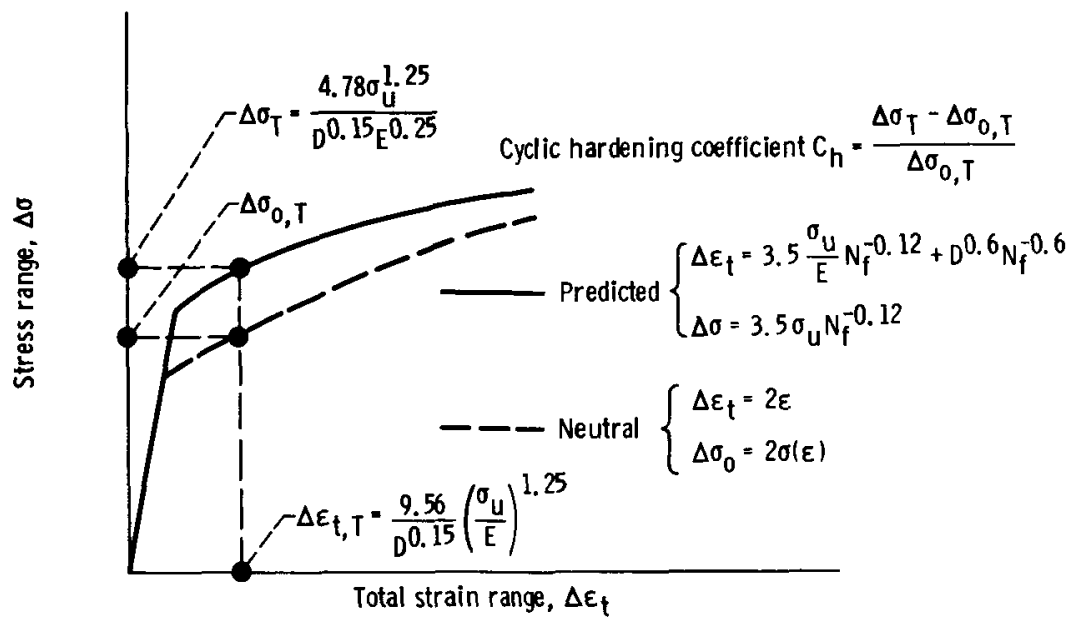

(a) Calculation procedure.

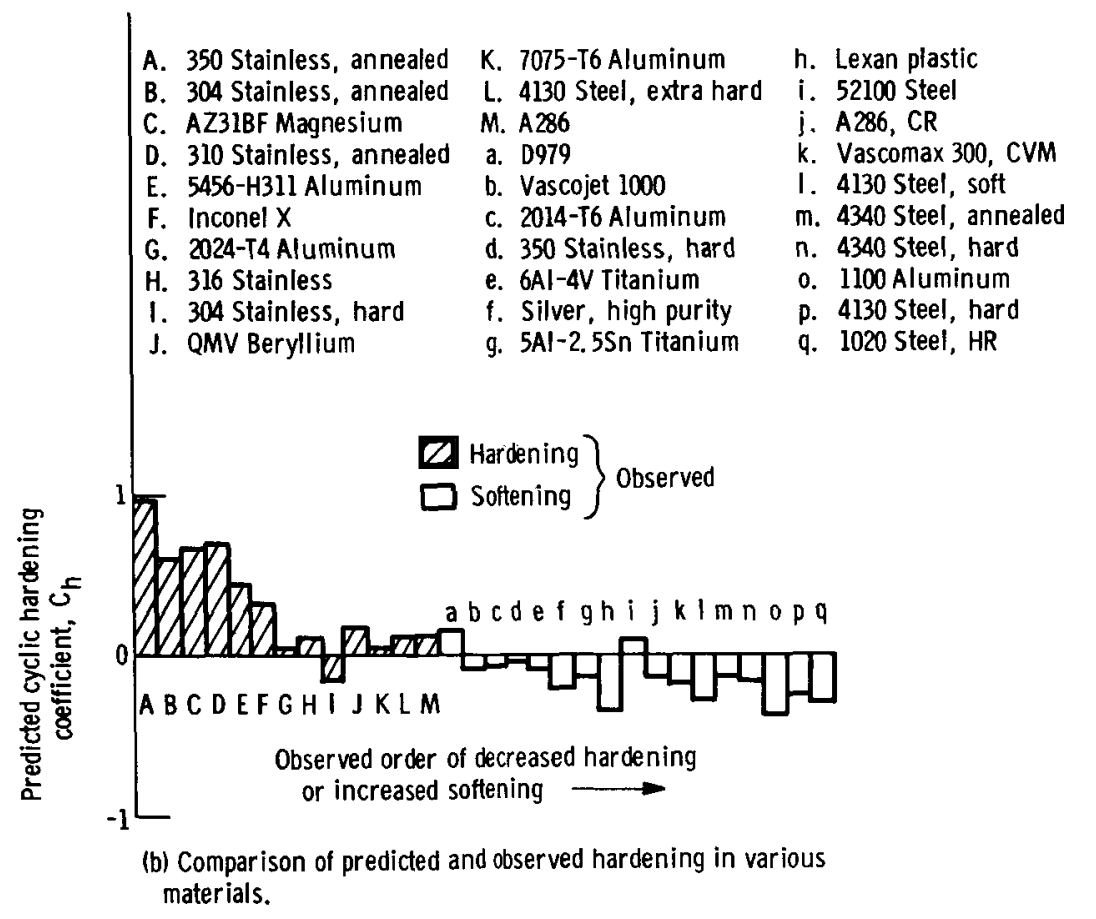

figure 5. - Calculation of cyclic strain hardening. 


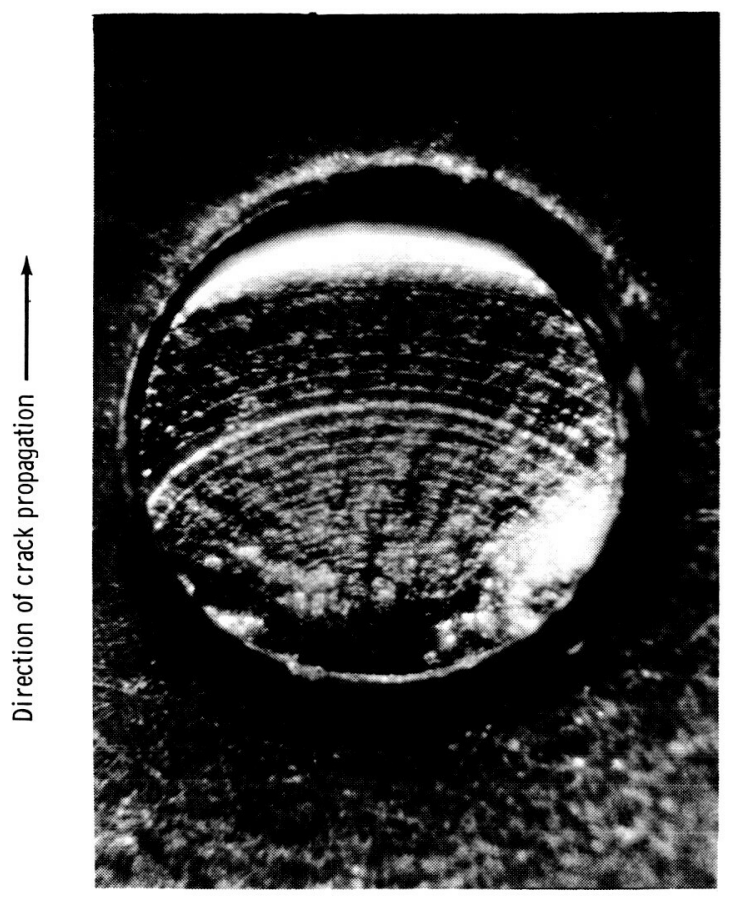

Figure 6. - Fracture surface of $1 / 4$ inch diameter copper specimen showing striations. $\mathrm{N}_{f}=\bar{I} \bar{u} \bar{u}$ cycies.

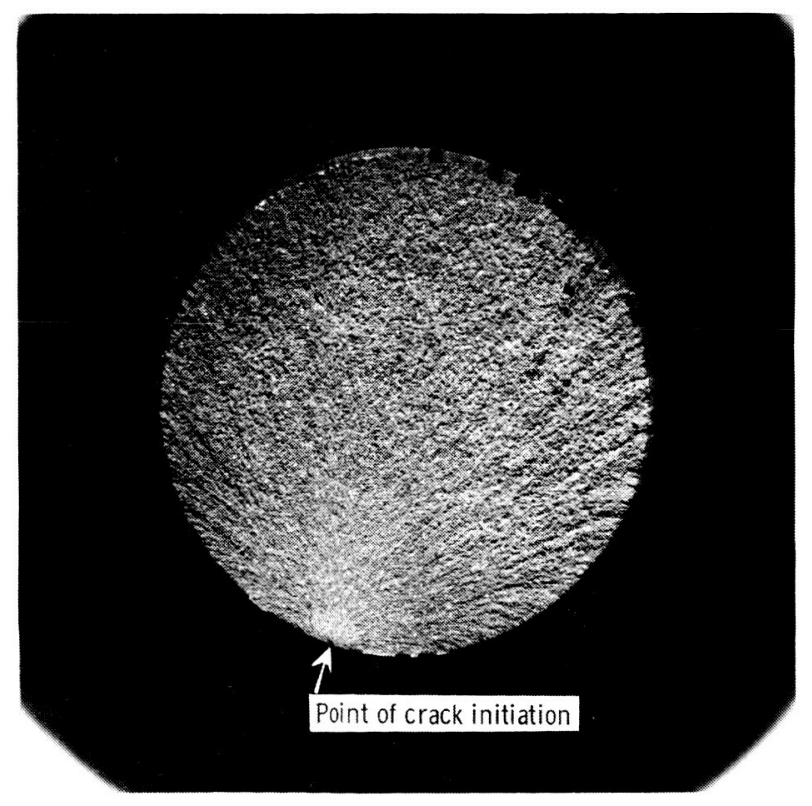

Figure 7. - Fracture surface of $1 / 4$ inch diameter $521001600^{\circ} \mathrm{F}$ temper) specimen. $N_{f}=10,030$ cycles. 
옹
N
1
ต1

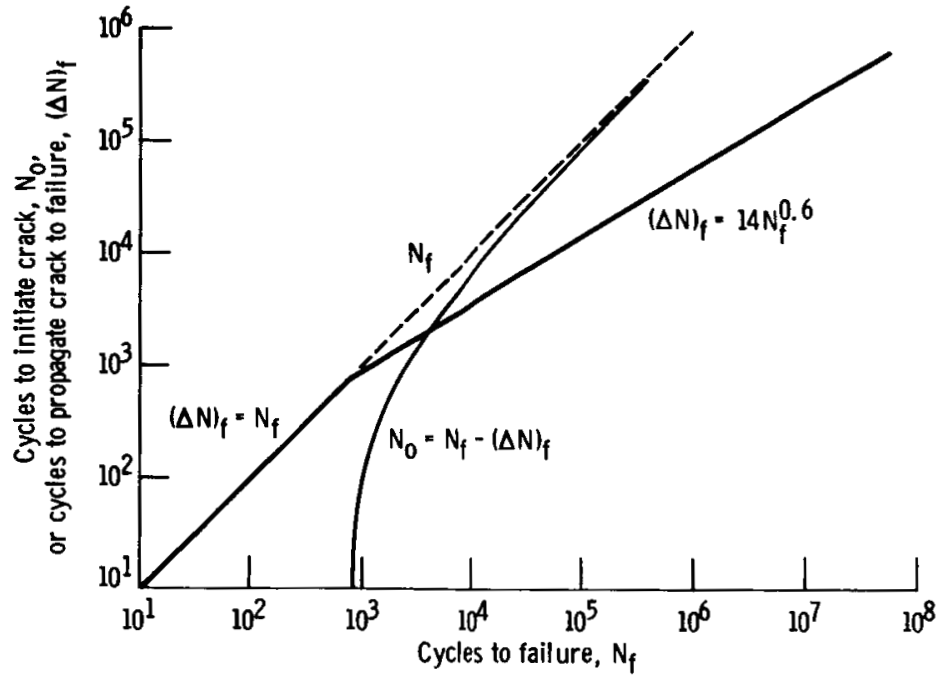

Figure 8. - Relation of crack initiation to crack propagation and failure.

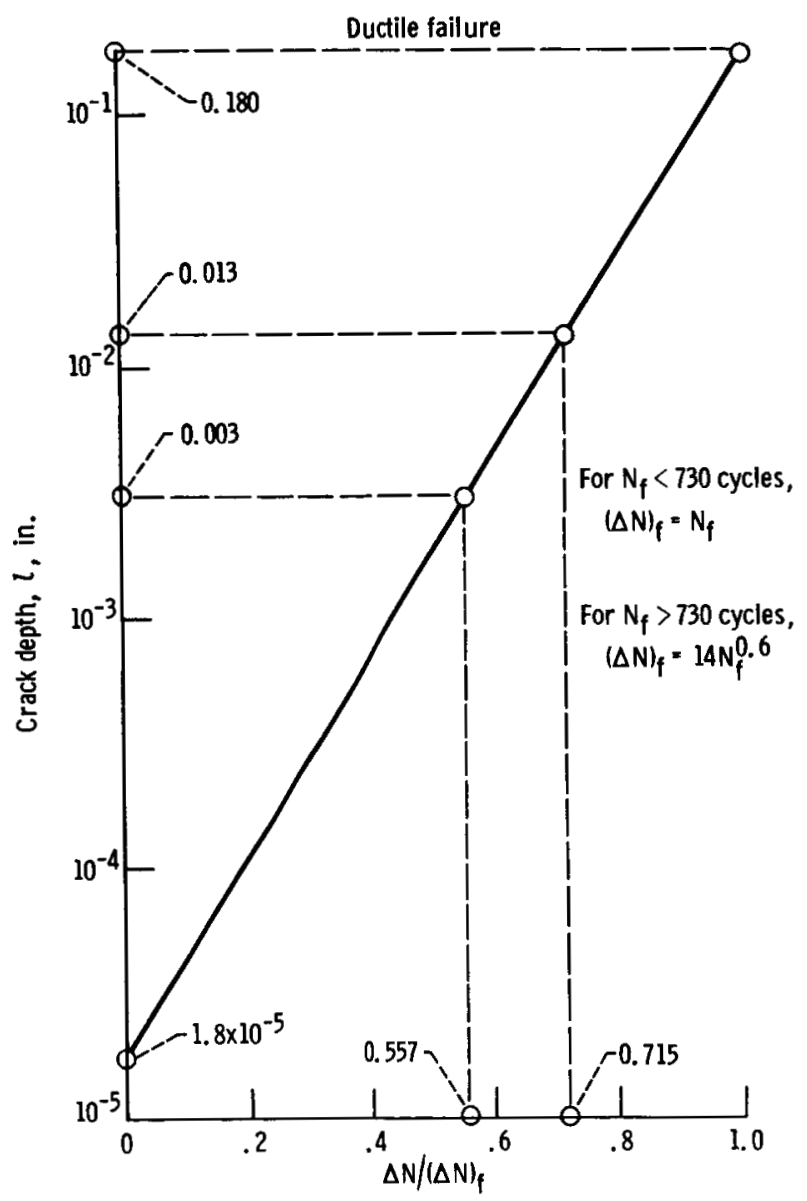

Figure 9. - Hypothesized crack growth relation. 


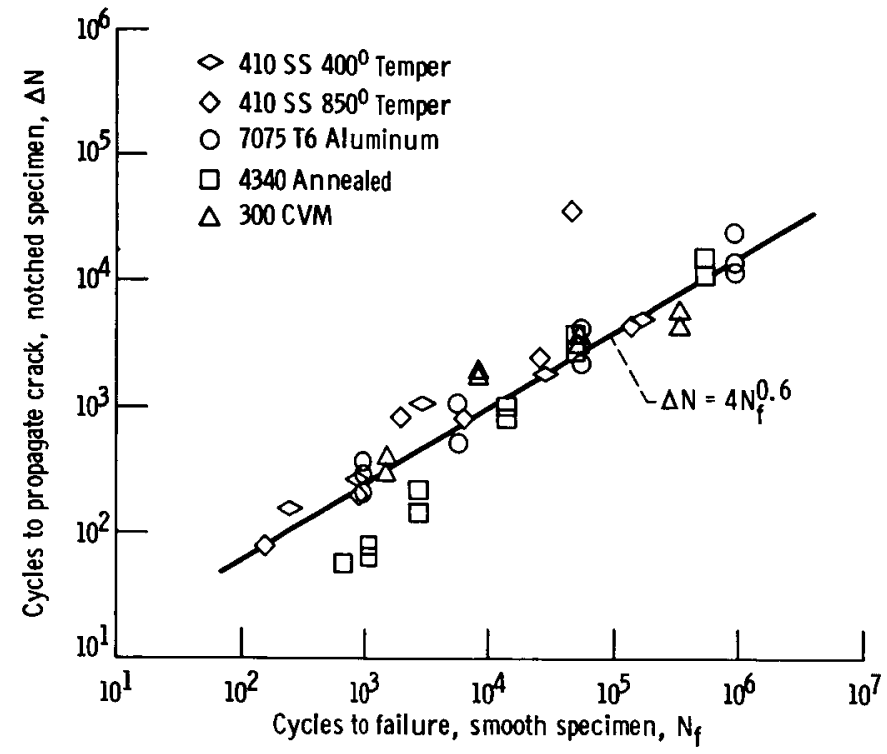

Figure 10. - Crack propagation for notched specimens.

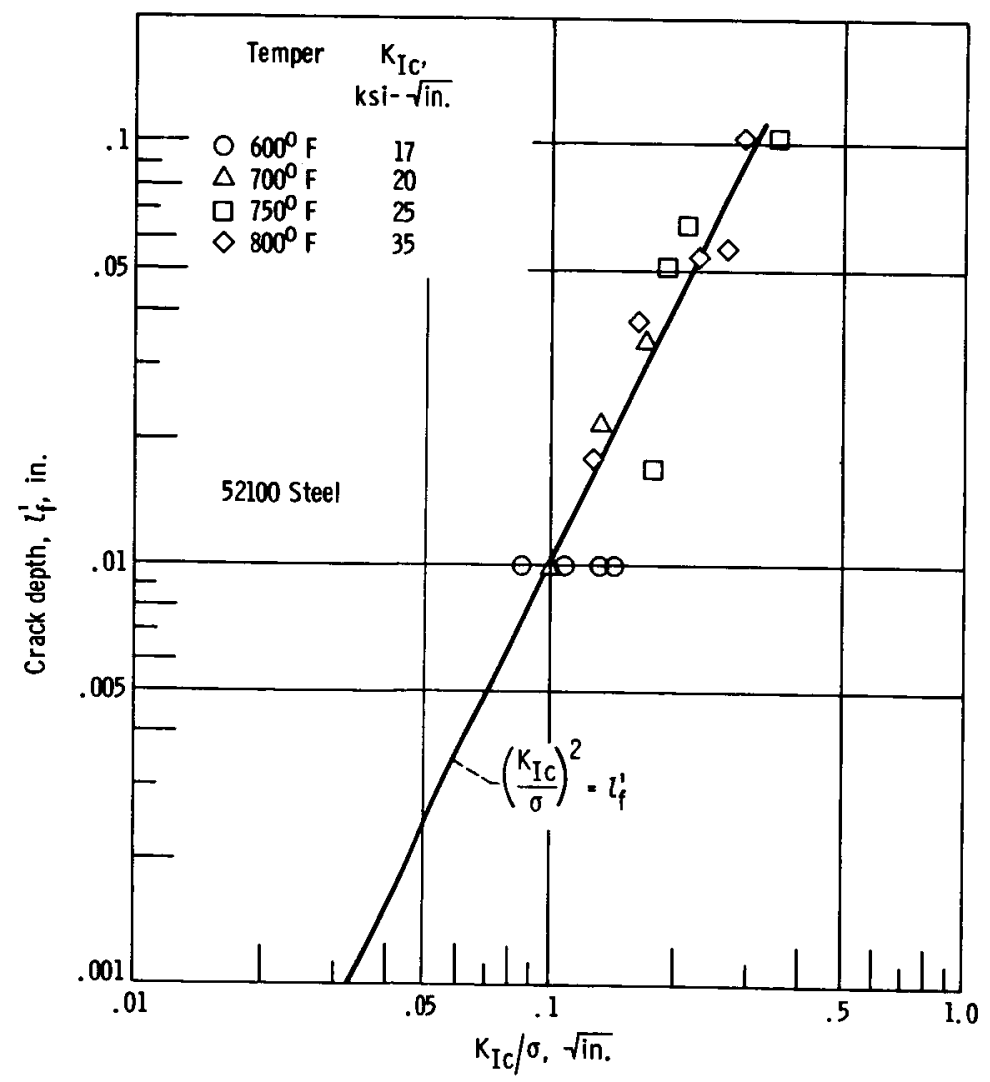

Figure 11. - Fracture relation. 


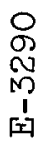

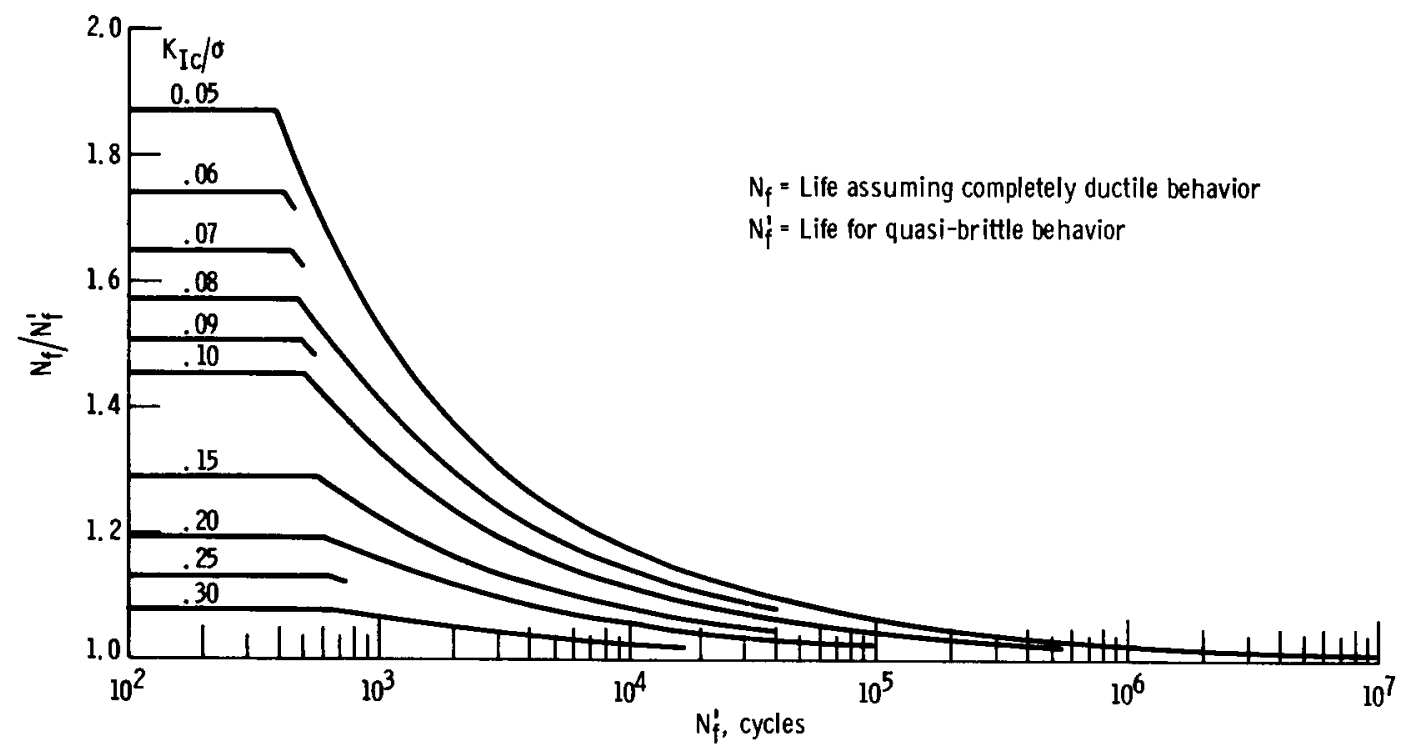

Figure 12. - Effect of notch toughness on life of smooth specimens. 

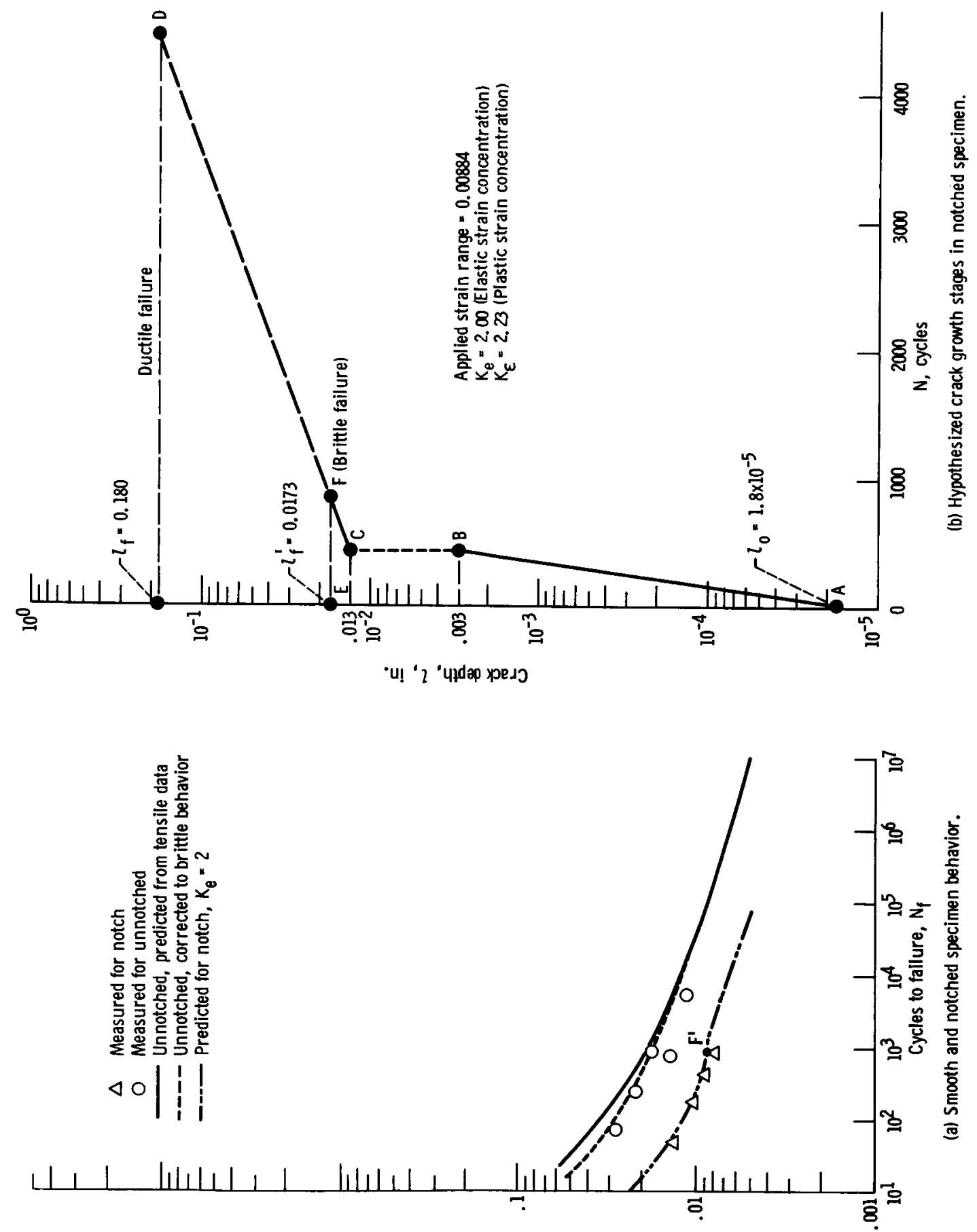

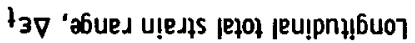


$L-605$
$N_{f}=70$

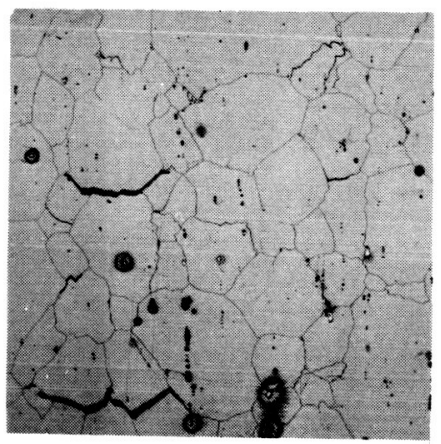

A -286

$N_{f}=38400$

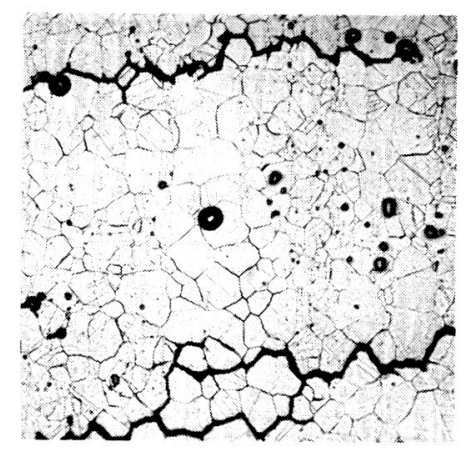

CS -37030

Figure 14. - Intergranular fatigue cracks at $1200^{\circ} \mathrm{F}$.

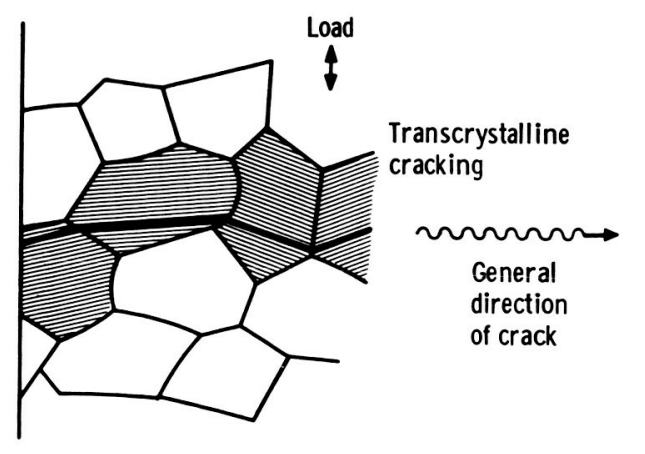

$N_{0}=N_{f}-6.2 N_{f}^{0.6}$

For $N_{f}=10^{4}$

$N_{0}=0.85 \times 10^{4}$

$\Delta \mathrm{N}=0.15 \times 10^{4}$

Total life $=1.0 \times 10^{4}$

(a) Below creep range.

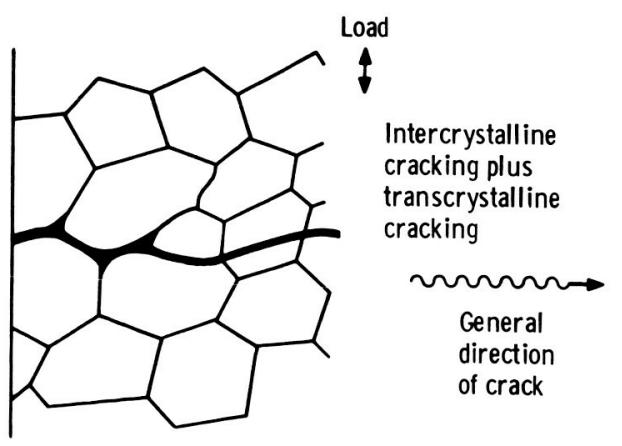

Assume $N_{0} \ll N_{f}-6.2 N_{f}^{0.6}$

At $\Delta \varepsilon$ for which $\left(N_{f}\right)_{\text {R.T. }}=10^{4}$

Limiting case, $\mathrm{N}_{0} \rightarrow 0$

$\Delta \mathrm{N}=0.15 \times 10^{4}$

Total life $=0.15 \times 10^{4}$

(b) In creep range.

Figure 15. - Illustration of high temperature crack initiation hypothesis. 


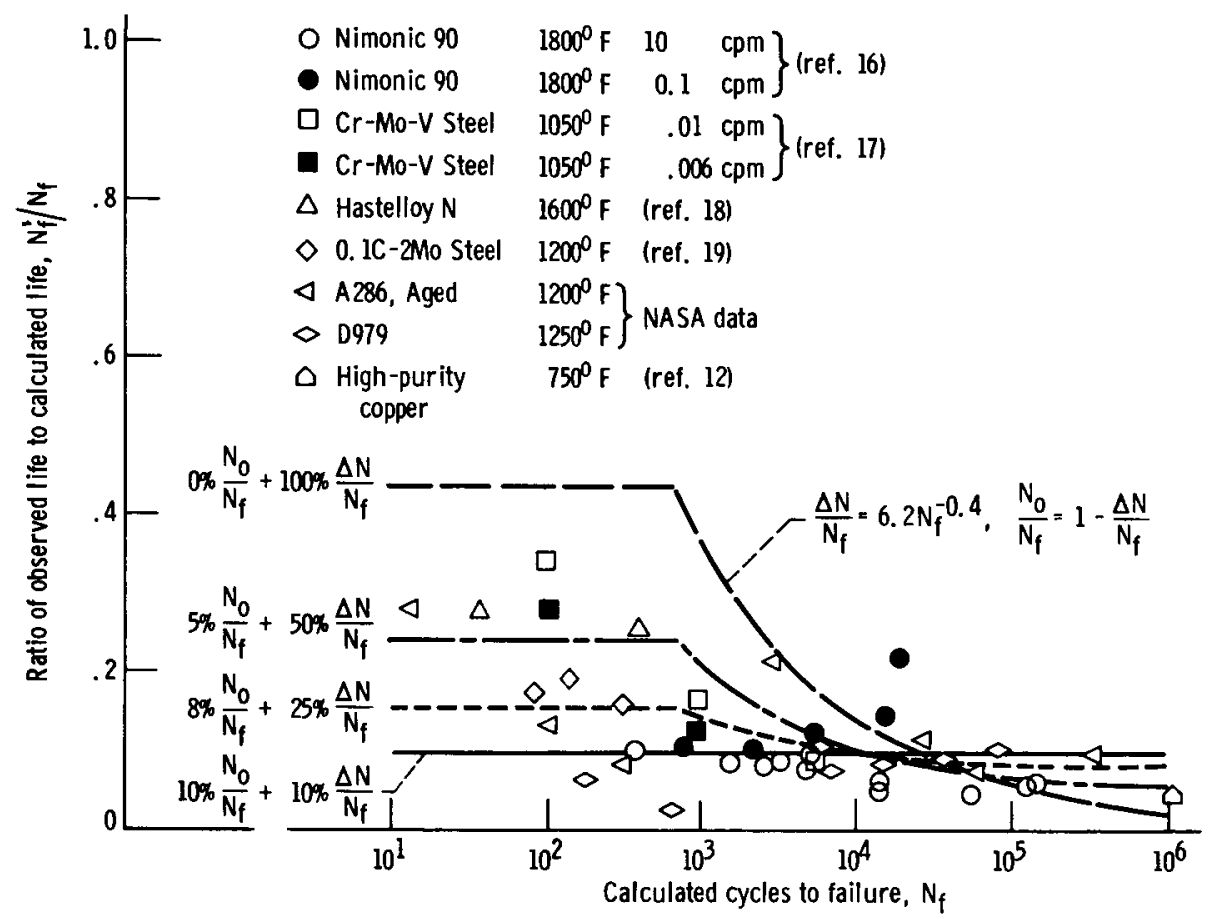

Figure 16. - High-temperature fatigue data and life-prediction curves. 


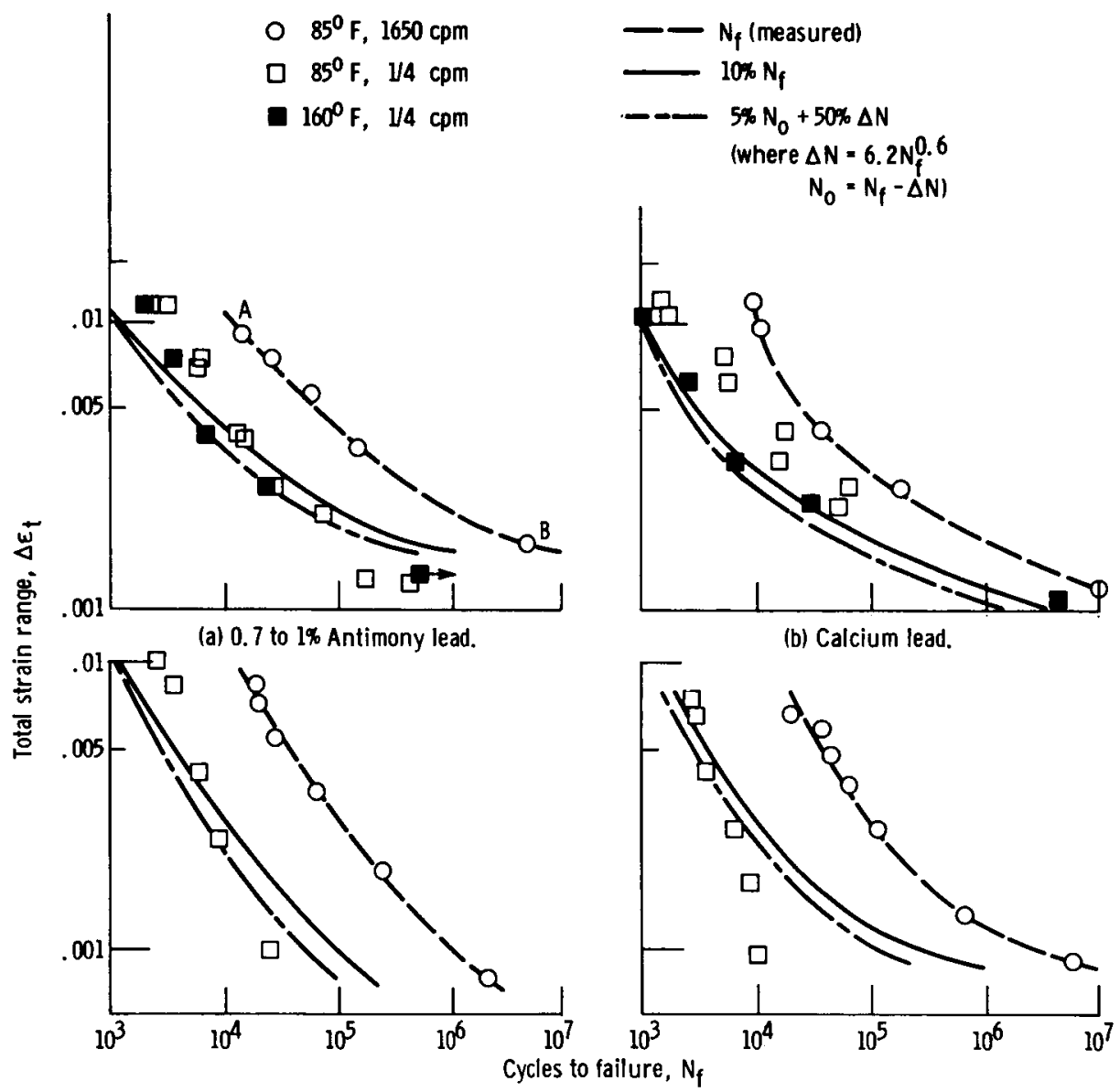

(c) Chemical lead.

(d) High purity lead.

Figure 17. - High-temperature fatigue hypothesis applied to lead (data from ref. 20 ). 


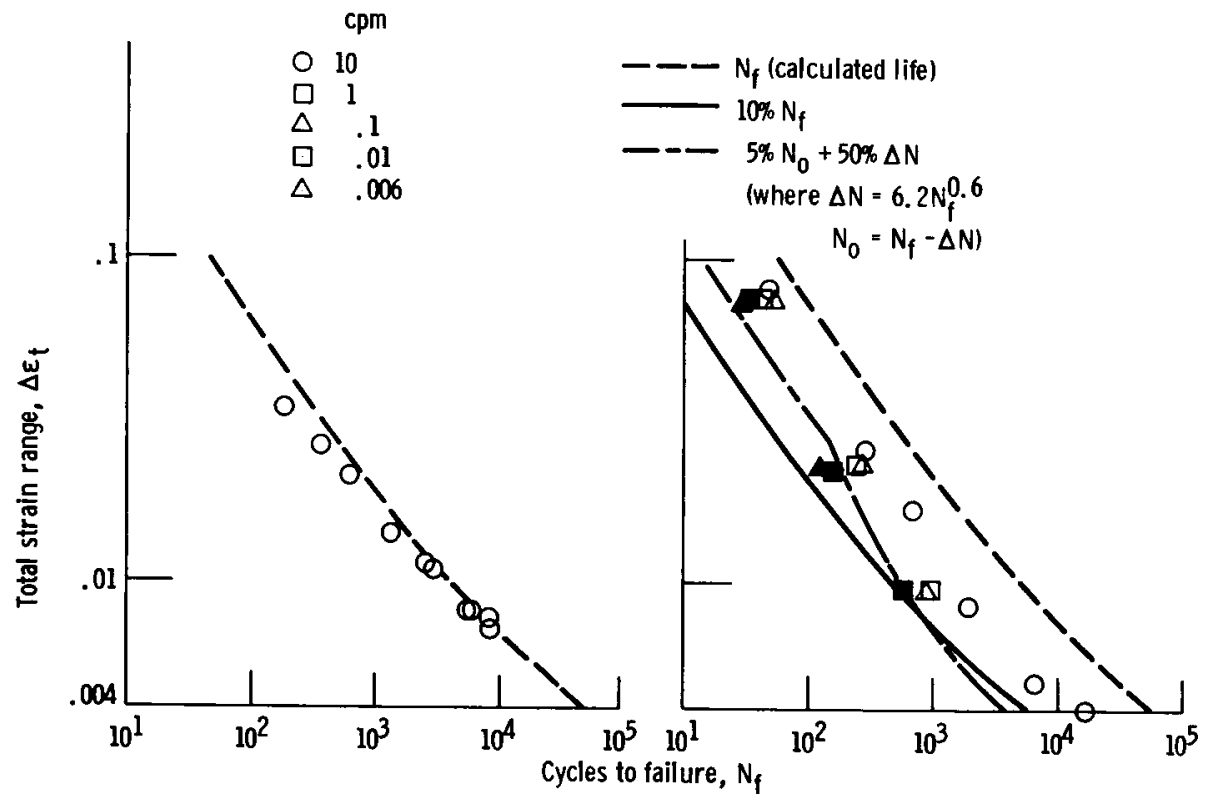

(a) Room temperature.

(b) $1050^{\circ} \mathrm{F}$.

Figure 18. - High-temperature hypothesis applied to $\mathrm{Cr}-\mathrm{M}_{0}-\mathrm{V}$ steel (data from ref. 17). 


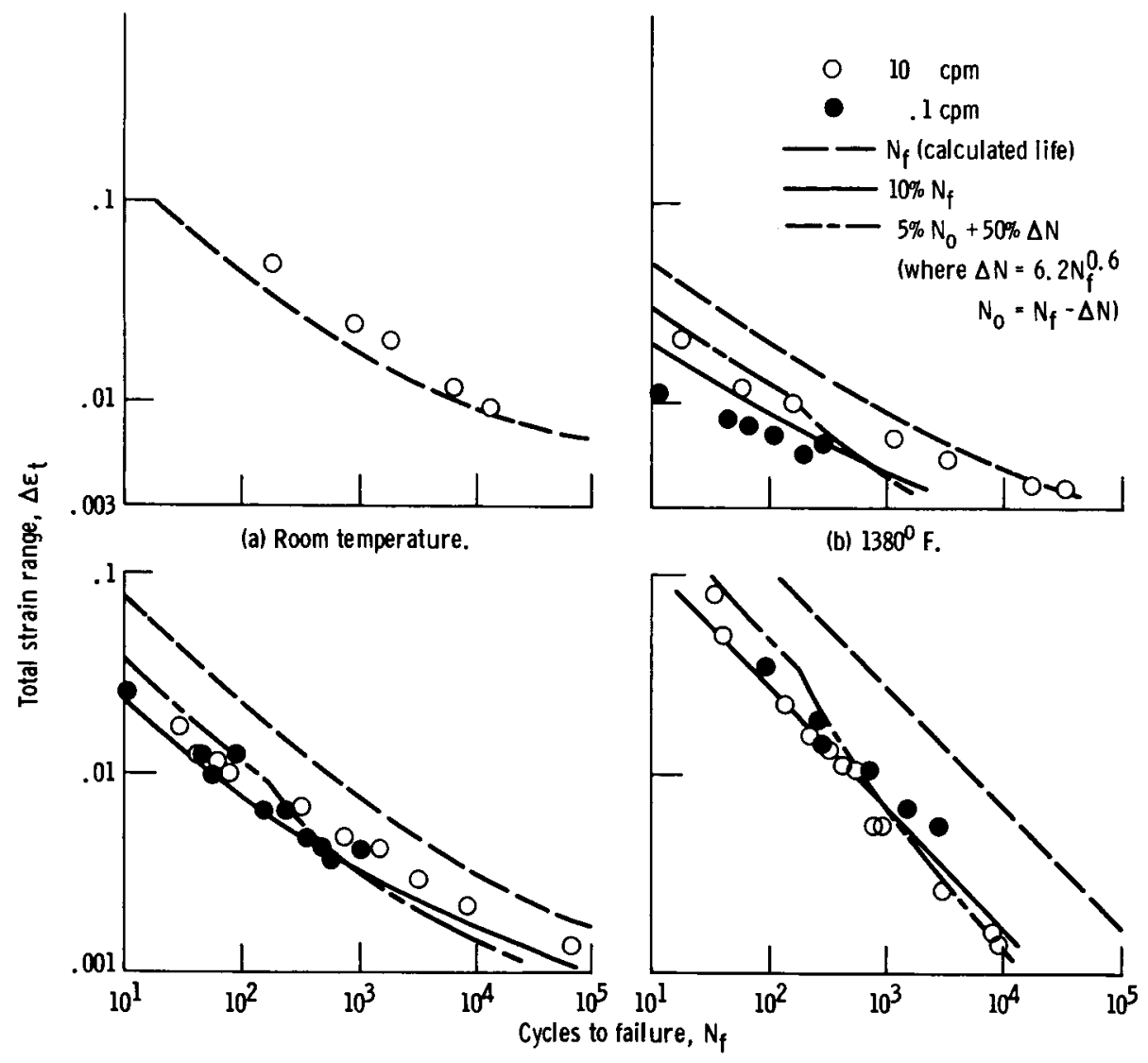

(c) $1600^{\circ} \mathrm{F}$.

(d) $1800^{\circ} \mathrm{F}$.

Figure 19. - High-temperature hypothesis applied to Nimonic 90 (data from ref. 16). 


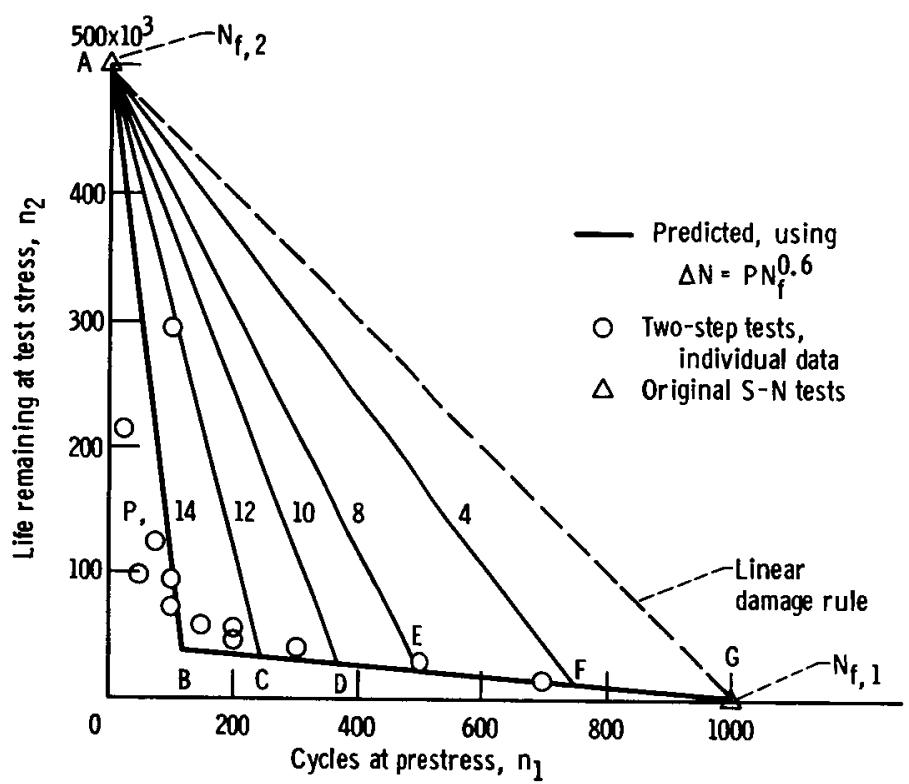

Figure 20. - Determination of crack propagation coefficient of maraging steel $(300 \mathrm{CVM})$ by two-step rotating-bending fatigue tests.

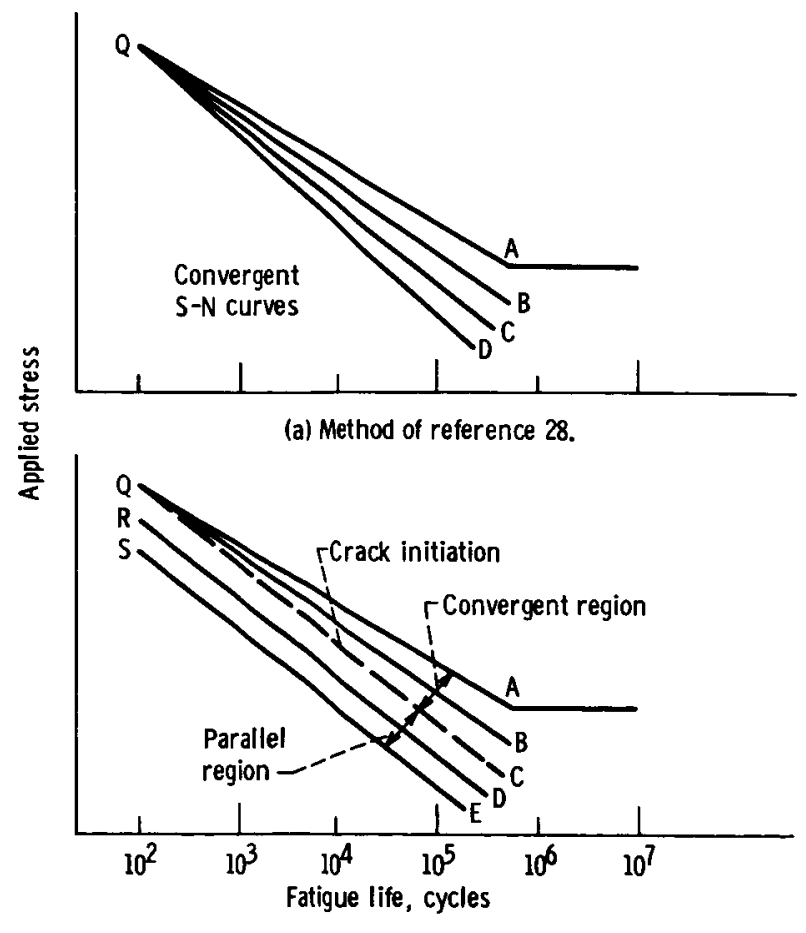

(b) Double linear damage hypothesis.

Figure 21. - Two methods of cumulative fatigue analysis. 


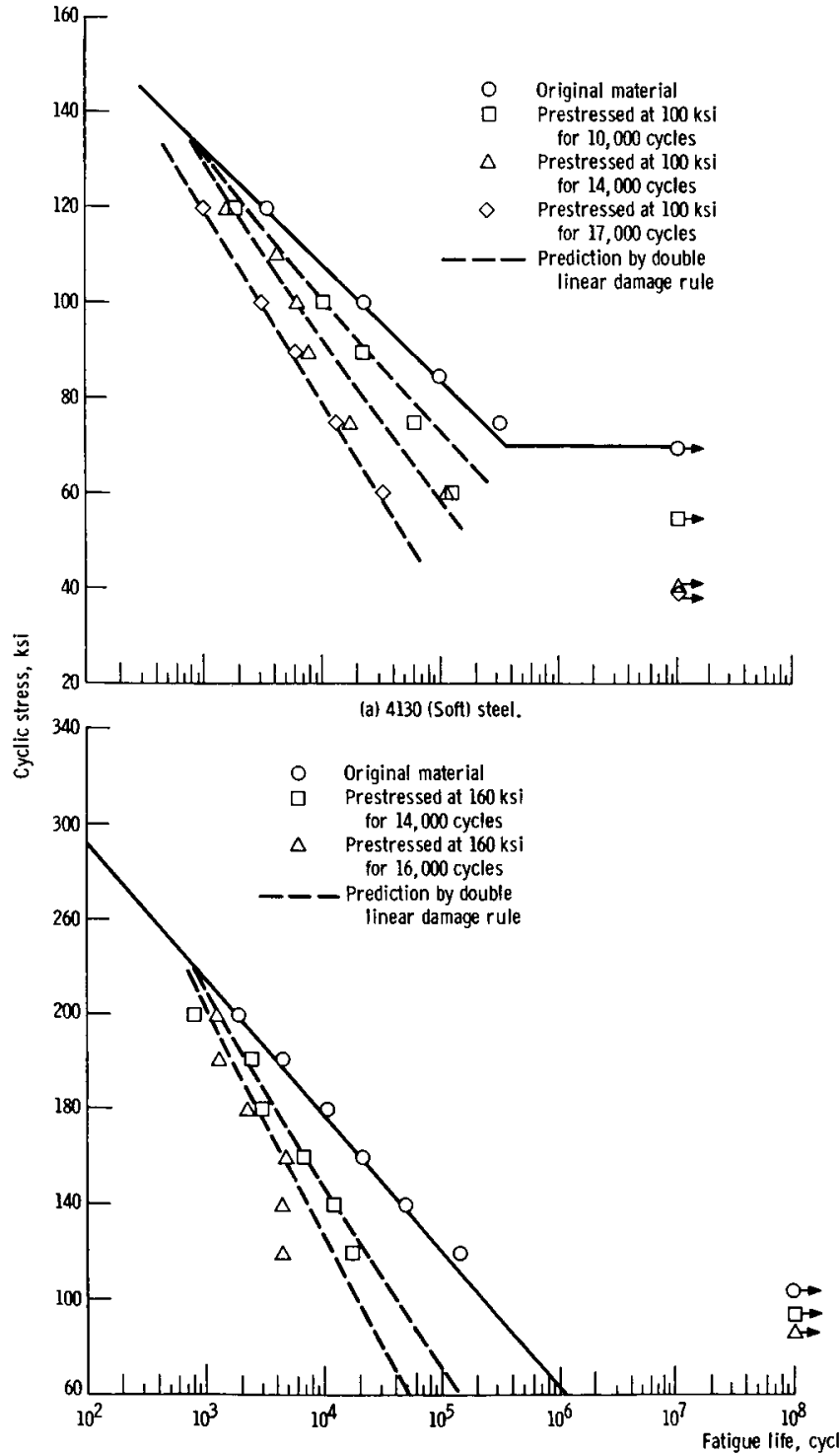

(c) 52100 Steel.
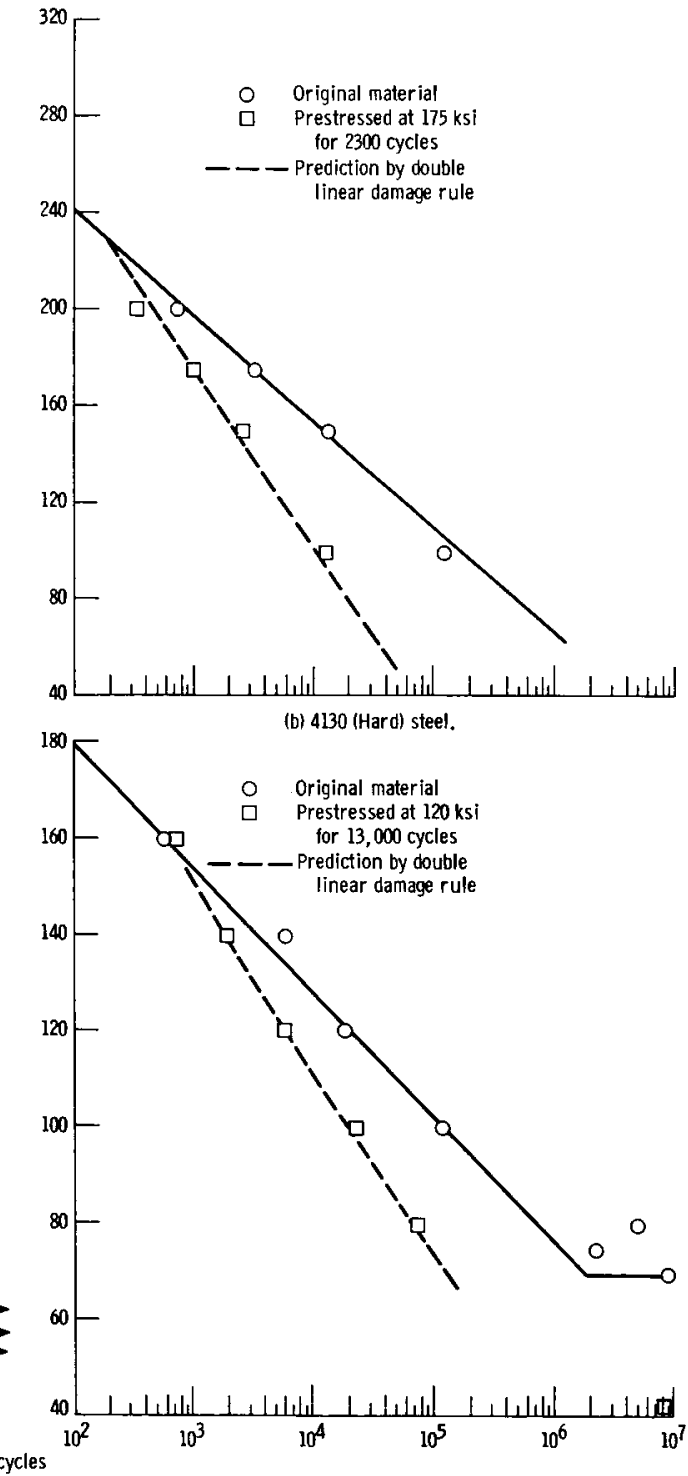

(d) 304 Stainless steel.

Figure 22. - Double linear damage rule applied to two-step fatigue data. 


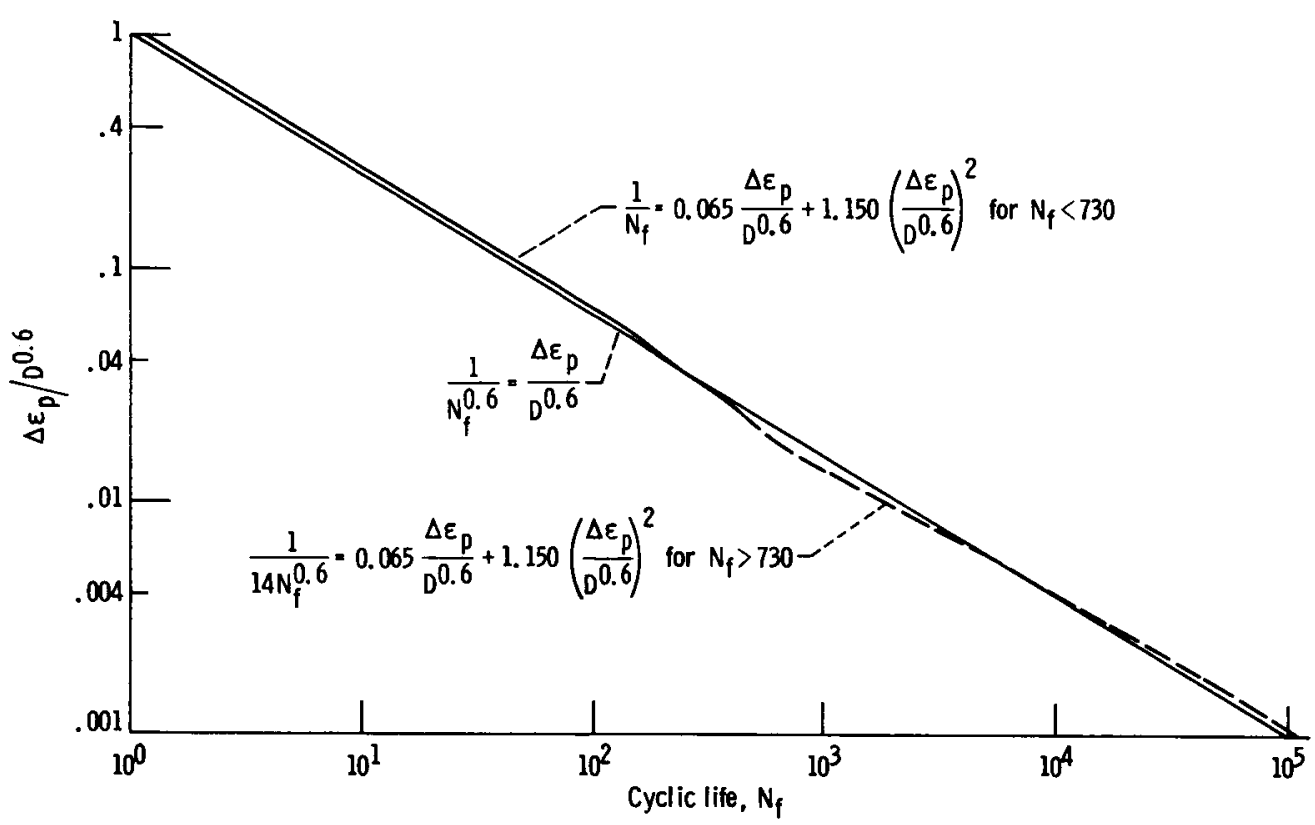

Figure 23. - Relation between cyclic life and plastic strain as derived from crack growth considerations and comparison with method of universal slopes. 